\title{
Contribution à l'étude du rapport au savoir en mathématiques de neuf professeurs des écoles expérimentés
}

Contribution to the study about nine primary school teachers' relation to mathematics

\section{Françoise Chenevotot, Marie-Pierre Galisson et Carole Baheux}

\section{OpenEdition Journals}

Édition électronique

URL : https://journals.openedition.org/educationdidactique/5110

DOI : 10.4000/educationdidactique.5110

ISSN : 2111-4838

Éditeur

Presses universitaires de Rennes

\section{Édition imprimée}

Date de publication : 30 décembre 2019

Pagination : 83-108

ISBN : 978-2-7535-8041-1

ISSN : 1956-3485

\section{Référence électronique}

Françoise Chenevotot, Marie-Pierre Galisson et Carole Baheux, « Contribution à l'étude du rapport au savoir en mathématiques de neuf professeurs des écoles expérimentés », Éducation et didactique [En ligne], 13-3 | 2019, mis en ligne le 05 février 2020, consulté le 10 octobre 2022. URL : http:// journals.openedition.org/educationdidactique/5110; DOI : https://doi.org/10.4000/ educationdidactique. 5110 


\title{
CONTRIBUTION À L'ÉTUDE DU RAPPORT AU SAVOIR EN MATHÉMATIQUES DE NEUF PROFESSEURS DES ÉCOLES EXPÉRIMENTÉS
}

\author{
Françoise Chenevotot \\ Laboratoire de didactique André Revuz (EA 4434), UA, UCP, UPD, UPEC, URN, \\ Université d'Artois, COMUE et INSPE Lille Nord de France, France \\ Marie-Pierre Galisson \\ Laboratoire de didactique André Revuz (EA 4434), UA, UCP, UPD, UPEC, URN, \\ Université d'Artois, COMUE et INSPE Lille Nord de France, France \\ Carole Baheux \\ Laboratoire de Mathématiques de Lens (EA 2462), Université d'Artois, France
}

Lesévaluations, tant nationales qu'internationales, soulèvent des interrogations sur l'enseignement des mathématiques en France. Le plus souvent, les professeurs de l'enseignement élémentaire (élèves de 6 à 11 ans) ne sont pas des spécialistes de la discipline "mathématique ». C'est pourquoi nous faisons l'hypothèse que le rapport au savoir mathématique scolaire des professeurs des écoles a une influence sur leurs pratiques déclarées et sur les enjeux qu'ils attribuent à l'enseignement de cette discipline. Notre étude, ciblée sur neuf enseignants de cycle 3 (élèves de 9 à 11 ans), cherche à appréhender le rapport au savoir en mathématiques de ces professeurs des écoles (PE). Notre échantillon n'est pas représentatif de la population ordinaire des PE : les neuf PE interrogés sont expérimentés; notre échantillon comprend une proportion importante de scientifiques (7 PE sont titulaires d'un Baccalauréat scientifique et 4 d'entre eux ont une formation scientifique d'au moins deux ans après le Baccalauréat); 6 PE interrogés sont maîtres formateurs et donc engagés dans la formation. Nous nous inscrivons dans le cadre théorique de l'approche socio-anthropologique du rapport au savoir élaboré par Charlot (1997) que nous avons adapté au contexte de notre recherche. Notre méthodologie s'appuie sur des entretiens semi-directifs. Nos analyses nous permettent de dégager quelques spécificités de ce rapport au savoir et leurs impacts sur les pratiques déclarées. Nous concluons sur quelques perspectives en termes de formation continue.

Mots-clés : rapport au savoir, professeurs des écoles, mathématiques.

Contribution to the study about nine primary school teachers' relation to mathematics

Both national and international assessments raise questions about the teaching of mathematics in France. In most cases, teachers in elementary schools (pupils from 6 to 11 years old) are not specialists in mathematics. That's why we assume that the relationship to the school mathematical knowledge of the primary school teachers has an influence on their declared practices and on the importance they give to this teaching. Our study, targeted at nine teachers of cycle 3 (pupils from 9 to 11 years old), seeks to focus on the relationship to mathematical knowledge of these primary school teachers. Our sample is not representative of the ordinary primary school teachers' population: the nine primary school teachers surveyed are experienced; our sample includes a significant proportion of scientists ( 7 of them hold a scientific French Baccalauréat and 4 of them have a scientific background of at least two years after the French Baccalauréat; six of them are teacher trainers and are therefore engaged in training. Our action takes part in the socio-anthropological approach to relationship 
to knowledge theoretical framework defined by Charlot (1997) which we adapted to the context of our research. Our methodology is based on semi-structured interviews. Analysis allows us to identify some specificities of this relation to knowledge and their impact on declared practices. We conclude with some perspectives for teacher in-service training.

Keywords : relation to knowledge, primary school teachers, mathematics.

Cette recherche a bénéficié du soutien financier de l'ESPE LNF en 2015 et 2016 suite à un appel à projet.

\section{INTRODUCTION}

Les rapports de l'Inspection Générale (MEN 2013) mettent en évidence un certain nombre de constats sur la pratique des professeurs des écoles (PE) en mathématiques: des domaines négligés (grandeurs et mesures) et des activités insuffisamment mises en œuvre (résolution de problèmes). Le fait que des activités soient éludées dans les séances d'enseignement soulève la question du sens, de la valeur, que leur confèrent les professeurs des écoles. Un rapport récent (MEN 2018, p. 6) ravive cette question : «Un tiers des professeurs des écoles déclare ne pas aimer enseigner les mathématiques ».

Appréhender le rapport au savoir en mathématiques des professeurs des écoles peut fournir un outil d'intelligibilité pour mieux comprendre les activités d'apprentissage proposées par ces professeurs en classe. Tel que le caractérise Charlot (1997, p. 93), le rapport au savoir est « une relation de sens, et donc de valeur, entre un individu (ou un groupe) et les processus ou produits de savoir $»$. Peu de recherches, jusqu'à présent, ont été consacrées au lien entre le rapport au savoir en mathématiques et les pratiques d'enseignement des professeurs des écoles (Magendie 2004).

Les dernières évaluations TIMSS 2015 (MEN 2016) soulignent encore deux aspects. D’une part, les enseignants français sont plus souvent mal à l'aise que leurs pairs européens pour enseigner les mathématiques et les sciences. D'autre part, au cours des deux années précédant l'étude, moins d'un enseignant sur deux a reçu une formation continue en mathématiques et seul un quart d'entre eux a bénéficié d'une formation continue en sciences. Une meilleure connaissance du rapport au savoir en mathématiques des PE permettrait de cibler les inter- ventions les plus opportunes en formations initiale et continue alors que de nouveaux programmes accompagnés d'une réorganisation pédagogique des cycles ont été mis en œuvre à la rentrée 2016 en France.

Nous avons choisi de "penser par cas ", c'est-àdire « de procéder par l'exploration et l'approfondissement des propriétés d'une singularité accessible à l'observation " pour "fonder une description, une interprétation, une évaluation » (Passeron $\&$ Revel 2005 , p. 9). Nous ne cherchons pas à recueillir des généralités sur les pratiques enseignantes déclarées des PE mais à analyser les choix que quelques PE interrogés déclarent faire en fonction de leur rapport au savoir mathématique scolaire. Notre échantillon, constitué de neuf PE enseignants en cycle 3, n'est pas représentatif de la population ordinaire des PE : les PE interrogés sont tous expérimentés; notre échantillon comprend une proportion importante de sujets ayant suivi une filière scientifique considérée à l'époque de leur scolarité lycéenne comme une filière ouverte à tous les choix d'orientation professionnelle (7 PE sont titulaires d'un bac scientifique et 4 d'entre eux ont une formation scientifique d'au moins deux ans après le bac) ; 6 PE interrogés sont maîtres formateurs et donc engagés dans la formation.

Notre étude exploratoire poursuit donc l'objectif de dégager des éléments d'intelligibilité pour appréhender le rapport au savoir en mathématiques des professeurs des écoles. Elle fait suite à une recherche analogue portant sur le rapport au savoir en physique de professeurs des écoles (Decroix \& Kermen 2016). Nous retenons les questions de recherche suivantes: - Peut-on trouver des spécificités du rapport au savoir mathématique scolaire de professeurs des écoles expérimentés?

- Comment ces éventuelles spécificités influencent-elles les pratiques déclarées d'ensei- 
gnement des mathématiques de ces professeurs?

Nous présentons les caractéristiques de notre contexte d'étude. Nous décrivons ensuite le cadre théorique que nous avons retenu, inspiré de l'approche socio-anthropologique du rapport au savoir défini par Charlot. Notre méthodologie s'appuie sur des entretiens semi-directifs enregistrés puis analysés selon une grille élaborée pour étudier le rapport au savoir en physique des professeurs des écoles (Decroix \& Kermen 2016). Après nos analyses, nous faisons un point sur nos questions de recherche puis proposons quelques éléments sur les perspectives en particulier en ce qui concerne la formation continue.

\section{LE SAVOIR MATHÉMATIQUE SCOLAIRE}

\section{Les mathématiques scolaires au cycle 3}

Les programmes officiels de 2008 pour le cycle 3 (CE2, CM1 et CM2) en vigueur au moment de notre étude constituent la référence principale des professeurs des écoles en termes de savoir mathématique scolaire.

\section{Prédominance du numérique parmi les quatre domaines}

En préambule, les concepteurs des programmes de 2008 (MEN 2008, p. 22) lient la « pratique des mathématiques » et le développement de compétences transversales " goût de la recherche et du raisonnement, l'imagination et les capacités d'abstraction, la rigueur et la précision » et concluent que la maîtrise des principaux éléments de mathématiques constitue une aide pour agir dans la vie quotidienne et poursuivre des études au collège.

Ces principaux éléments (thèmes d'études) sont répartis en quatre domaines :

1. Nombre et calcul : les systèmes de nombres élargis au système des nombres décimaux et à une approche des nombres rationnels; le calcul mental, posé et à la calculatrice. La résolution de problèmes liés à la vie courante contribue à donner sens aux nombres et aux algorithmes.

2. Géométrie : le recours aux instruments pour mettre en évidence des propriétés et des objets reconnus perceptivement doit permettre aux élèves de passer d'une géométrie de la perception à une géométrie instrumentée qui s'appuie sur des propriétés. Les problèmes de reproduction et de construction sont des leviers de ce passage.

3. Grandeurs et mesures: les grandeurs usuelles avec comme nouveauté les aires et les volumes, les unités de grandeurs usuelles et les mesures associées, quelques formules (périmètres, aires, volumes). Les problèmes « concrets » contribuent à donner sens à ces connaissances et compétences.

4. Organisation et gestion de données : le concept de proportionnalité et les notions qui en dérivent (pourcentage, échelle, conversion, agrandissement et réduction de figures); des outils novateurs (la règle de trois) sont introduits en termes d'outils de résolution. La résolution de problèmes issus de la vie courante ou " tirés d'autres enseignements » permet de travailler ce domaine.

Les compétences du socle ${ }^{1}$ (palier 3 ) telles que, par exemple, « utiliser et construire des tableaux, des diagrammes, des graphiques et savoir passer d'un mode d'expression à un autre » doivent s'inscrire dans une progression à la charge du professeur. Cette progression peut s'appuyer sur un synoptique qui décline les diverses connaissances et compétences selon les trois niveaux CE2, CM1, CM2 qui composent le cycle 3 au moment de notre étude.

La répartition horaire de l'enseignement des mathématiques en cycle 3 est fixée à 180 heuresannée par niveau, à savoir cinq heures par semaine. Cette répartition sur l'année peut être adaptée s'il y a mise en œuvre d'un projet pédagogique.

La place du numérique est prépondérante dans les programmes scolaires de 2008 et l'ordre de présentation des domaines coïncide avec l'étendue de la description de ces programmes. Historiquement (d'Enfert 2003, 2015), les mathématiques de l'école primaire ont été caractérisées par la prédominance de l'arithmétique (nombres, calculs, problèmes concrets) au détriment de la géométrie dont la légitimité s'ancrait dans l'utilisation du système métrique. Les nombreuses réformes qui ont modelé les programmes depuis les années 1970 ont délégitimé le domaine des grandeurs et mesures (perte des liens entre numération et système métrique), le domaine de la géométrie (la géométrie ne relevant plus que de la leçon de choses et de vocabulaire) pour rétablir progressivement (depuis 1995) la pertinence épistémologique des quatre domaines en vigueur en 2008. 


\section{Place de la résolution de problèmes}

Pour les programmes de 2002 (et avant), « la résolution de problèmes est au centre des activités mathématiques » (MEN 2002, p. 82) et un document d'accompagnement des programmes (les problèmes pour chercher) a proposé une typologie des problèmes ${ }^{2}$ (MEN 2005, p. 7). Les programmes de 2008 ont réaffirmé le rôle privilégié des problèmes « la résolution de problèmes joue un rôle essentiel dans l'activité mathématique » tout en laissant les PE plus libres pour la mise en œuvre de la démarche " elle est présente dans tous les domaines et s'exerce à tous les stades des apprentissages » (MEN 2008, p. 38). En dehors de ses caractérisations contextuelles « problèmes liés à la vie courante », " problèmes de reproduction ou de construction », " problèmes concrets », " problèmes de la vie courante ou tirés d'autres enseignements » (MEN 2008, p. 23), la résolution de problèmes n'est plus explicitement liée à ses fonctions : construire de nouveaux savoirs, les appliquer, les consolider, développer des stratégies de recherche.

\section{La transposition didactique} des mathématiques scolaires

L'enjeu des mathématiques scolaires au cycle 3 a fortement évolué entre le moment où les PE ont été formés et celui où ils enseignent. Le rapport institutionnel de certains objets mathématiques change $\mathrm{du}$ fait des évolutions curriculaires. Ceci induit ( $a$ priori) des modifications dans le rapport personnel des enseignants à ces objets mathématiques. Et cela n'est pas sans incidence sur la transposition didactique qu'ils doivent opérer pour enseigner les mathématiques scolaires.

Le découpage des programmes en vigueur en 2008 rend compte des "modifications qui concernent à la fois les mathématiques savantes, les pratiques de la vie courante pour le mesurage et l'école» (Chambris 2010, p. 319). Les travaux de Chambris témoignent que, si les grandeurs, les nombres et les opérations ont toujours été enseignés à l'école primaire, leurs relations ont évolué depuis la réforme des mathématiques modernes. En substituant à l'arithmétique, deux domaines, le numérique et la mesure, la réforme écarte une théorie de référence transposée à l'enseignement, « une théorie des grandeurs qui pilote le numérique » (Chambris
2010, p. 331). Elle la remplace par une théorie du numérique (toujours en vigueur selon l'auteur) « qui gouverne l'enseignement des nombres, des opérations et de la proportionnalité; le " numérique », c'est-à-dire ici, une théorie des nombres non entiers fondée sur les entiers et une théorie des fonctions linéaires numériques» (Chambris 2010, p. 328). De fait, " les conditions de vie des objets impliquant grandeurs, nombres ou opérations ont été dégradées au moment de la réforme; depuis cette époque, leur vie est ainsi fragilisée malgré divers remaniements » (Chambris 2010, p. 325). L'auteur signale les difficultés des élèves actuels, par exemple en numération, et relève l'absence dans l'enseignement primaire de problèmes de numération de la vie courante du type « trouver le nombre de paquets de 100 carreaux qu'il faut acheter pour avoir 8564 carreaux » (Chambris 2010, p. 323). Les travaux de Chambris (2010) tendent à légitimer la nécessité de concevoir un savoir de référence, une théorie éclairant les relations entre grandeurs, nombres et opérations destinées à être transposées à l'enseignement. « Le but principal est d'assurer la possibilité de rendre interprétable dans la théorie choisie les discours des élèves et donc de proposer aux enseignants un cadre pour les interpréter» (Chambris 2010, p. 362).

La résolution de problèmes soulève les mêmes questions de transposition. Disqualifiant le découpage d'un enseignement de la résolution de problèmes, rabattu préalablement sur la lecture et le traitement de l'information (ce que peuvent suggérer les programmes et les manuels antérieurs à 2002), les travaux de Houdement (2003) mettent en évidence que « La résolution de problèmes n'est pas dissociable de la maîtrise des connaissances mathématiques nécessaires pour les résoudre. [...] Il n'existe pas de méthodologie générale de résolution, chacun recourt à une mémoire personnelle des problèmes. Cette mémoire des problèmes est une mémoire de schémas de problèmes (Julo 2002); c'est elle qui facilite le processus essentiel de structuration. Elle se forme à partir des différents problèmes que nous rencontrons, des représentations que nous en construisons et des analogies que nous percevons " (Houdement 2003, p. 19). Servir à construire et maîtriser des connaissances mathématiques, proposer une multiplicité de situations où ces connaissances sont mobilisées pour favoriser leur disponibilité, est la finalité formulée dans les programmes de 2002 et plus implicitement ceux de 2008 de la résolution de problèmes. 
Ces évolutions traduisent un nouveau rapport institutionnel à la résolution de problèmes. Il faut donc être attentif à la perception que les PE ont ou pas de ces évolutions en termes de rapport au savoir.

\section{Une spécificité du rapport au savoir} des professeurs des écoles

Le rapport au savoir mathématique scolaire du professeur des écoles se distingue a priori de celui du professeur de mathématiques dans l'enseignement secondaire. En tant qu'élève, l'attirance disciplinaire initiale qui est souvent à l'origine de l'orientation du professeur de l'enseignement primaire porte fréquemment sur les sciences herméneutiques plutôt que sur les sciences formelles et empirico-formelles (Develay 1995). Le professeur des écoles a la charge explicite d'opérer une harmonisation interdisciplinaire, de penser un développement conjoint des apprentissages dans les diverses disciplines, ce qui n'est pas le cas pour le professeur de mathématiques. De même, l'harmonisation intradisciplinaire (interprétation des programmes, des enjeux de la discipline) peut prendre en compte une approche plus holistique de la genèse des concepts mathématiques et se distingue de l'harmonisation intra-disciplinaire pratiquée par le professeur de mathématiques qui élabore des organisations mathématique et didactique du savoir à enseigner en fonction de ses finalités disciplinaires.

\section{CADRE THÉORIQUE}

\section{Différentes approches du rapport au savoir}

Depuis plus de trente ans, plusieurs équipes françaises de chercheurs en sciences de l'éducation et en didactique des disciplines se sont intéressées à la notion de rapport au savoir (Caillot 2014). Trois principales approches, que nous allons présenter brièvement, ont émergé : (1) l'approche clinique de Beillerot, Mosconi et Blanchard-Laville, (2) l'approche socioanthropologique de Charlot, Bautier et Rochex et (3) l'approche anthropologique de Chevallard.

Dans l'approche clinique proposée par Beillerot \& al (1996), d'inspiration psychanalytique, le rapport au savoir se définit comme la disposition d'un sujet (élève, enseignant...) envers le savoir et a la particu- larité de mettre en jeu l'histoire entière du sujet : sa façon de savoir, d'apprendre et son désir de savoir. Si le rapport au savoir est lié au désir de savoir, il ne s'y limite donc pas car le savoir n'apparaît que comme un objet de désir parmi d'autres, distinct et extérieur au sujet.

L'approche socio-anthropologique élaborée par Charlot (1997) est une sociologie du sujet (élève) qui a été développée pour questionner l'échec scolaire. Les chercheurs sont entrés dans des classes de milieux défavorisés pour comprendre ce qui posait problème aux élèves dans leurs apprentissages. Ils ont pour cela observé des écarts et des divergences entre les rapports au savoir des élèves au moyen de bilans de savoir et d'entretiens semi-directifs. Pour ces chercheurs, le rapport au savoir est un rapport à l'apprendre qui s'inscrit dans l'histoire personnelle et scolaire du sujet. Nous détaillerons dans le paragraphe suivant.

Dans l'approche anthropologique du rapport au savoir proposée par Chevallard (2003), les institutions et les personnes jouent un rôle important. L'institution est un dispositif social qui impose à ses sujets des manières spécifiques de faire et de penser. L'individu, par le fait de ses assujettissements à de nombreuses institutions, se constitue en une personne (ensemble formé par l'individu et les divers rapports personnels qu'il entretient avec les objets). Le rapport personnel, qui émerge d'une pluralité de rapports institutionnels, ne va pas de soi car les manières de faire au sein de l'institution peuvent créer des tensions avec les rapports personnels du sujet à certains objets.

Pour notre étude, nous avons retenu l'approche socio-anthropologique élaborée par Charlot car elle rend possible une perspective didactique $\mathrm{du}$ rapport au savoir et permet de questionner le sens des apprentissages en s'intéressant au sujet apprenant. De plus, comme nous l'avons signalé dans l'introduction, nous nous inscrivons dans un projet de recherche plus vaste visant à comparer le rapport au savoir en mathématiques et en physique de professeurs des écoles (Decroix \& al, 2019). C'est pourquoi, nous avons privilégié un cadre d'analyse commun, l'approche socio-anthropologique, qui fonde la recherche antérieure portant sur le rapport au savoir en physique des PE (Decroix \& Kermen 2016). Nous allons maintenant présenter cette approche plus précisément. 


\section{Approche socio-anthropologique} du rapport au savoir

Pour Charlot (1997, p. 34) «Étudier le rapport au savoir, c'est étudier ce sujet (l'élève) en tant qu'il est confronté à la nécessité d'apprendre et à la présence dans le monde de savoir ». Car pour Charlot (1997, p. 98) « naître, c'est entrer dans un monde où l'on est obligé d'apprendre ». En stipulant que « le sujet apprend [...] en s'engageant dans une activité » (Charlot 2003, p. 48), et que « pour qu'il y ait activité, il faut que l'enfant se mobilise » (Charlot 1997, p. 61), l'auteur caractérise la mobilisation du sujet dans l'activité par son investissement, son « usage de soi comme d'une ressource ", sa mise « en mouvement par des mobiles qui renvoient à du désir, du sens, de la valeur » (Charlot 1997, p. 63).

Charlot (1997, p. 67) précise encore que « la question de «l'apprendre » est plus large que celle du savoir » et « qu'il faudrait (donc) distinguer le « rapport à l'apprendre » (forme la plus générale) du « rapport au savoir » (forme spécifique du « rapport à l'apprendre ») ». Plus précisément, pour Charlot (1997, p. 94), « le rapport au savoir est l'ensemble (organisé) des relations qu'un sujet entretient avec tout ce qui relève de "l'apprendre " et du savoir ». Charlot (1997, p. 73) pose qu'« il n'y a de sujet de savoir et il n'y a de savoir que dans un certain rapport au monde - qui se trouve être, en même temps, un rapport au savoir ». Dans cette approche, le rapport au savoir présente une dimension épistémique (nature de l'acte d'apprendre et du fait de savoir) et une dimension identitaire où « apprendre fait sens en référence à l'histoire du sujet, à ses attentes, à ses repères, à sa conception de la vie, à ses rapports aux autres, à l'image qu'il a de lui-même et à celle qu'il veut donner aux autres » (Charlot 1997, p. 84-85). Ces deux dimensions sont traversées par une dimension sociale car le sujet est inscrit dans un espace social.

\section{Adaptation aux enseignants de l'approche socio-anthropologique du rapport au savoir}

Bien que ce cadre ait été développé pour les élèves, plusieurs recherches se sont appuyées sur l'approche socio-anthropologique pour caractériser le rapport au savoir d'enseignants.

\section{Au niveau de l'enseignement primaire}

Magendie (2004) s'intéresse au rapport au savoir et à son influence sur les pratiques ordinaires d'enseignement de deux PE. Elle interroge les liens entre le rapport à l'apprendre d'un enseignant, son rapport à l'apprendre en mathématiques et les modalités d'apprentissage qu'il propose et permet à ses élèves pendant une séance de mathématiques. Sa recherche l'a conduite à analyser "l'enseignant-apprenant * sous le prisme de "l'enseignant-élève " (l'élève que l'enseignant se souvient d'avoir été) (Magendie 2004, p. 112). Elle montre que le type d'activité que les PE proposent est lié au rapport que ces PE entretenaient avec les savoirs disciplinaires lorsqu'ils étaient élèves.

Pautal, Venturini et Dugal (2008) étudient des pratiques d'enseignement et d'apprentissage en SVT. Ils s'appuient sur le modèle de l'action didactique conjointe de Sensevy et sur l'approche socio-anthropologique du rapport au savoir pour analyser les interactions entre le professeur et les élèves. Les auteurs analysent les rapports au savoir d'une enseignante et de quelques élèves de CM2 durant une séance de biologie sur la circulation sanguine. Ils identifient deux types de rapport au savoir en opposition. Si pour les élèves, les savoirs en jeu sont privilégiés pour les explications scientifiques qu'ils apportent, pour l'enseignante, les aspects transversaux priment sur les contenus de savoirs.

Maizières (2011) s'intéresse au rapport au savoir musical des PE et plus spécifiquement au transfert entre les propres compétences de l'enseignant et ses pratiques d'enseignement. La problématique du rapport au savoir musical des PE est posée dans le cadre proposé par Charlot même si l'auteur souligne que celui-ci permet de définir le « rapport à l'apprendre » mais pas son « rapport à l'enseigner » (Maizières 2011, p. 3). Malgré cette limite, Maizières utilise les dimensions identitaire et épistémique, ainsi que les caractéristiques des processus épistémiques du savoir identifiées par Charlot pour faire émerger différentes catégories de pratiques enseignantes à partir d'interviews portant sur 16 PE. Ces diverses catégories illustrent la complexité du processus de transfert entre le rapport au savoir musical de l'enseignant et ses pratiques d'enseignement et mettent en évidence l'impact d'un rapport identitaire au savoir à enseigner (le sens de cet enseignement). 


\section{Au niveau de l'enseignement secondaire}

Mornata (2015) fonde ses recherches sur l'hypothèse que le rapport au savoir, de nature éminemment sociale, se manifeste par un contenu, des croyances sur le savoir et l'apprentissage, issus d'un processus de construction caractérisé par un investissement identitaire. L'auteure s'appuie sur le cadre élaboré par Charlot et sur les théories de l'épistémologie personnelle. Elle caractérise le rapport au savoir comme un processus dynamique entre des dimensions épistémique (constituée de croyances sur le savoir et l'apprentissage), identitaire (le savoir étant un moyen de se définir) et sociale (sa nature étant historico-culturelle). La méthodologie est basée sur des interviews menées auprès de 19 enseignants de diverses disciplines dans une même école (élèves de 12 à 15 ans) en Suisse et porte sur le rapport au savoir en général sans lien explicite avec un domaine didactique. Mornata (2015, p. 84) valide son hypothèse de recherche : "Le rapport au savoir est un processus dynamique entre des dimensions épistémique, identitaire et sociale. Il se manifeste par des croyances sur le savoir issues d'un processus d'investissement identitaire».

Quelles que soient les méthodologies de recueil de données, nous retenons que ces travaux témoignent des potentialités de l'approche socio-anthropologique pour mettre à jour des liens entre le rapport au savoir des enseignants et, d'une certaine façon, sous certains aspects, leur rapport à l'enseigner.

\section{Autres recherches sur le rapport au savoir des enseignants}

Signalons encore une recherche qui, bien que consacrée à l'étude du rapport au savoir de l'enseignant et de l'apprenant, ne s'appuie pas sur l'approche socio-anthropologique. Les travaux de Calmettes concernent l'enseignement secondaire et font référence à des didactiques disciplinaires. Calmettes (2015) s'intéresse à la démarche d'investigation pour l'enseignement scientifique pour laquelle il met en perspective les rapports au savoir des enseignants et les rapports pragmatiques à l'enseigner. Le rapport au savoir est étudié d'une part dans le cadre anthropologique de Chevallard et d'autre part grâce à la modélisation pragmatiste de l'action didac- tique de l'enseignant de Calmettes. L'auteur prend aussi en compte la théorie de la valuation (Dewey). Il identifie trois idéaltypes des rapports au savoir grâce à des questionnaires soumis à 82 enseignants de physique débutants et à une étude de cas d'enseignants chevronnés.

L'étude quantitative et qualitative de Calmettes, centrée sur des pratiques, permet d'identifier trois idéaltypes ciblés sur la démarche d'investigation en partie liée à la démarche de résolution de problèmes que nous cherchons à caractériser. Nous retenons, mais à une échelle différente, sa méthodologie de recueil de données même si nous ne nous inscrivons pas dans son cadre d'analyse.

Par ailleurs, des approches anglo-saxonnes en psychologie cognitive ont défini le concept de croyances épistémologiques "epistemic beliefs » (élève, enseignant...) qui présente des liens avec la notion francophone de rapport au savoir et lui fait écho. En s'appuyant sur l'approche socio-anthropologique et les croyances épistémologiques, Therriault, Morel et Letscher (2015) ont interrogé le rapport aux savoirs que les futurs professeurs de sciences et de sciences humaines entretiennent et ils ont identifié leurs croyances épistémologiques relatives à la nature de la connaissance dans l'objectif d'établir des relations avec les conceptions de l'enseignement et de l'apprentissage.

Les travaux de Therriault ont la particularité de s'appuyer sur deux cadres théoriques: d'une part le cadre francophone de rapport au savoir et d'autre part le cadre anglo-saxon des croyances épistémologiques. Cette approche s'est avérée porteuse pour étudier le rapport au savoir de futurs enseignants québécois et a permis de mettre en évidence l'impact des croyances épistémologiques (apparentées à la dimension épistémique du rapport au savoir) sur les conceptions de l'enseignement. Toutefois, si notre étude a débuté avant les travaux de Therriault cités, leurs résultats peuvent faire écho avec certains de nos propres constats.

\section{ÉVOLUTION DU CADRE THÉORIQUE}

Nous faisons l'hypothèse qu'il est possible d'éclairer les pratiques déclarées d'enseignement des professeurs des écoles en mathématiques grâce au concept de rapport au savoir élaboré par Charlot. Par cette entrée, nous pensons appréhender la mobilisation 
des PE à s'engager dans des activités ayant pour finalité l'enseignement des mathématiques mais nécessitant d'apprendre pour enseigner. Compte tenu du caractère large des définitions formulées par Charlot (1997), nous proposons une manière particulière pour adapter (catégories prédéfinies avant analyse) les dimensions épistémique et identitaire du rapport au savoir aux individus spécifiques que sont les PE en posture d'enseignant.

\section{Dimension épistémique}

Pour reprendre Charlot (1997, p. 78), «Apprendre, c'est avoir quel type d'activité? Analyser ce point, c'est travailler le rapport au savoir en tant que rapport épistémique ». Charlot définit les « figures de l'apprendre » et dresse « l'inventaire des figures sous lesquelles se présentent du savoir et de « l'apprendre » (1997, p. 77). Ainsi, « les enfants sont confrontés à la nécessité d'apprendre en rencontrant, dans un monde qui est déjà là », lors de l'acte d'apprendre :

- des objets-savoirs, c'est-à-dire des objets dans lesquels est incorporé du savoir : livres, monuments et œuvres d'art, émissions télévisées « culturelles »...;

- des objets qu'il faut apprendre à utiliser, des plus familiers (brosse à dents, lacets...) aux plus élaborés (appareil photo, ordinateur...); - des activités à maîtriser, de statut divers: lire, nager, démonter un moteur...;

- des dispositifs relationnels où entrer et des formes relationnelles à s'approprier, qu'il s'agisse de dire merci ou d'engager une relation amoureuse (Charlot 1997, p. 77).

De la même façon, nous faisons l'hypothèse que les enseignants sont confrontés à la nécessité d'enseigner en rencontrant dans un monde (qui est déjà là), lors de l'acte d'enseigner :

- des objets-savoirs : livres (de l'élève, du maître), programmes scolaires, textes de savoirs disciplinaires scolaires, textes de savoirs didactiques;

- des objets qu'il faut apprendre à utiliser : tableau, vidéoprojecteur, TBI, matériel pédagogique et didactique (rapporteur, boîtes à compter...);

- des activités à maîtriser : créer un climat de travail propice aux apprentissages, gérer l'organisation pédagogique (alternance de différentes modalités de travail individuel / collectif, passation des consignes, institutionnalisation), mettre en ouvre une démarche pédagogique (résolution de problèmes, démarche d'investigation);

- des relations à prendre en compte lors de l'acte d'enseignement : interactions avec les élèves (étayage, relations de respect, d'empathie, d'autorité si nécessaire), avec les familles, entre pairs et avec la hiérarchie.

Mettre en parallèle des objets concernant l'acte d'apprendre (pour l'élève) et l'acte d'enseigner (pour le PE) n'occulte pas que «le savoir et les figures de l'apprendre " (Charlot 1997, p. 67) ne sont pas de même nature. Ces figures de l'apprendre sont en résonance avec certaines catégories des connaissances mathématiques pour enseigner dégagées par Ball \& al (2008). Ainsi, ces auteurs déclinent les «Domains of Mathematical Knowledge for Teaching » en « Subject Matter Knowledge » et " Pedagogical Content Knowledge ». Un lien peut être fait entre « Subject Matter Knowledge » et les objets-savoirs ainsi qu'entre «Pedagogical Content Knowledge » et les activités à maîtriser. Nous ne détaillerons pas davantage les rapports entre ces deux approches car, plus qu'un état des lieux des connaissances déclarées ou observées des PE, c'est en termes de processus que nous cherchons à appréhender quelques spécificités de leur rapport au savoir épistémique.

Charlot (1997, p. 77-78) précise que « Face à ces objets, à ces activités, à ces dispositifs et formes, l'individu qui « apprend » ne fait pas la même chose, l'apprentissage ne passe pas par les mêmes processus ». Au cours de son histoire personnelle, l'individu qui enseigne s'est d'abord construit un rapport à l'apprendre dont Magendie (2004) souligne la survivance forte chez les enseignants qu'elle a étudiés. Ces rencontres passées avec des "figures de l'apprendre » (Charlot 1997, p. 67) ne peuvent être sans lien avec le rapport au savoir scolaire à enseigner que l'enseignant peut construire aujourd'hui. De même, face aux objets et aux activités citées, l'individu qui enseigne établit un rapport au savoir scolaire qui renvoie également à une diversité de processus d'apprentissage.

Nous supposons que les trois formes de rapport épistémique au savoir dégagées par l'approche socioanthropologique (Charlot 1997, p. 80-83) peuvent nous permettre de mieux comprendre la dimension 
épistémique du rapport à l'apprendre des PE (cf. tableau 1).

En restreignant l'usage de ce cadre théorique à un savoir spécifique que constituent les mathématiques scolaires et à des sujets particuliers qui sont des professeurs des écoles, nous sommes conscientes que la forte imbrication des trois formes de processus va limiter nos données et analyses.

Toutefois, cette interprétation nous conduit finalement à caractériser la dimension épistémique du rapport au savoir des PE en distinguant trois catégories d'un rapport à l'enseigner pour les mathématiques scolaires:

- le rapport à des savoirs disciplinaires scolaires qui peut se caractériser en termes de processus « d'objectivation-dénomination »; - le rapport à l'institution (programmes scolaires dont horaires dévolus aux différents enseignements) qui peut nous renseigner sur des processus en termes « d'imbrication du Je dans la situation » (l'activité engagée dans le monde est ici dans l'école telle que l'organise la société);

- le rapport à des ressources (dont les ressources matérielles) et des démarches pédagogiques et didactiques (dont les contraintes horaires inhérentes à ces démarches). Ce dernier rapport peut rendre compte de processus d'apprentissage en termes de « distanciationrégulation » (l'ensemble de ces ressources humaines ou matérielles qui permettent le contrôle du système relationnel du sujet en situation et en acte).

\section{Dimension identitaire}

En termes de rapport identitaire (Charlot 1997, p. 85), « apprendre fait sens en référence à l'histoire du sujet, à ses attentes, à ses repères, à sa conception de la vie, à ses rapports aux autres, à l'image qu'il a de lui-même et à celle qu'il veut donner aux autres » car « tout rapport au savoir est aussi rapport à soi-même [...] et également à l'autre».

Nous transposons les propos de Charlot aux enseignants pour lesquels enseigner fait sens en référence à l'histoire du sujet, à ses attentes, à ses repères, à sa conception de la vie, à ses rapports aux autres, à l'image qu'il a de lui-même et à celle qu'il veut donner aux autres.

Tout rapport au savoir est d'abord pour l'enseignant rapport à soi-même à travers l'acte d'enseigner. L'enseignant cherche à conquérir son autonomie et un statut dans la communauté de pratique. Son image positive d'enseignant est liée à la perception d'une certaine réussite professionnelle. Comme le signale

Tableau 1 : Trois formes de rapport épistémique au savoir dégagées par Charlot

\begin{tabular}{|c|c|c|}
\hline \multicolumn{2}{|c|}{ Cadre proposé par Charlot pour les élèves } & Adaptation aux professeurs des écoles \\
\hline $\begin{array}{l}\text { Le processus épis- } \\
\text { témique nommé } \\
\text { « objectivation-déno- } \\
\text { mination» }\end{array}$ & $\begin{array}{l}\text { Il « constitue, dans un même mouvement, } \\
\text { un savoir-objet et un sujet conscient de } \\
\text { s'être approprié un tel savoir », pour lequel } \\
\text { « apprendre, c'est passer de la non posses- } \\
\text { sion à la possession, de l'identification d'un } \\
\text { savoir virtuel à son appropriation réelle » } \\
\text { (Charlot 1997, p. 80). }\end{array}$ & $\begin{array}{l}\text { Il renvoie dans leur énonciation à des savoirs } \\
\text { disciplinaires dont la maîtrise des contenus } \\
\text { théoriques et didactiques en tant qu'enseignant } \\
\text { va de soi. }\end{array}$ \\
\hline $\begin{array}{l}\text { Le processus épisté- } \\
\text { mique « imbrication } \\
\text { du Je dans la situa- } \\
\text { tion » }\end{array}$ & $\begin{array}{l}\text { "Lapprendre est maîtrise d'une acti- } \\
\text { vité engagée dans le monde » pour lequel } \\
\text { apprendre c'est passer de « la non-maîtrise } \\
\text { à la maîtrise d'une activité » (Charlot 1997, } \\
\text { p. 81). }\end{array}$ & $\begin{array}{l}\text { L’apprendre réside dans la maîtrise de gestes } \\
\text { professionnels en conformité avec les exigences } \\
\text { de l'institution. }\end{array}$ \\
\hline $\begin{array}{l}\text { Le processus épisté- } \\
\text { mique « distanciation- } \\
\text { régulation » }\end{array}$ & $\begin{array}{l}\text { L'apprendre renvoie à la maîtrise d'une } \\
\text { relation en situation, ou à une énonciation } \\
\text { sous forme de règle, de principe pour lequel } \\
\text { apprendre c'est maîtriser une relation } \\
\text { (Charlot 1997, p. 83). }\end{array}$ & $\begin{array}{l}\text { L'apprendre renvoie à la maîtrise d'une relation } \\
\text { pédagogique (contrat pédagogique établi avec } \\
\text { les élèves et indépendant de la discipline) et du } \\
\text { contrat didactique (spécifique de la discipline } \\
\text { scolaire en jeu) instauré avec les élèves, les } \\
\text { ressources mobilisées en amont et en action pour } \\
\text { assurer le contrôle des situations. }\end{array}$ \\
\hline
\end{tabular}


Charlot (1997, p. 85) « On le sait, la réussite scolaire (c'est-à-dire la reconnaissance par l'institution et / ou la réussite des élèves, pour les enseignants) produit un puissant effet de réassurance et de renforcement narcissique et l'échec de gros dégâts dans la relation à soi-même ».

Tout rapport au savoir est aussi rapport à l'autre. Si, pour l'élève, " cet autre est celui qui m'aide à apprendre les mathématiques » (Charlot 1997 , p. 85), pour l'enseignant, cet autre est celui qui l'aide à enseigner. Il est « celui que j'admire ou que je déteste » et aussi ce "fantôme de l'autre que chacun porte en soi » (Charlot 1997, p. 85). En effet, « apprendre (et enseigner pour les enseignants) c'est toujours entrer dans un rapport avec l'autre, l'autre physiquement présent dans mon monde (c'est-à-dire, formateurs, collègues, inspecteur, élèves, parents... pour les enseignants), mais aussi cet autre virtuel que chacun porte en soi comme un interlocuteur » (Charlot 1997, p. 85).

\section{Dimension sociale}

Pour Charlot (1997, p. 87) « Cette dimension sociale ne s'ajoute pas aux dimensions épistémique et identitaire : elle contribue à leur donner une forme particulière ». Adaptée aux enseignants, la dimension sociale recouvre en partie ce rapport au monde qui les place en posture de professeur dans un espace social. Elle donne aussi consistance au sens et à la valeur que les PE octroient à ce savoir mathématique scolaire.

D'une part, elle peut renvoyer à l'utilité que les enseignants confèrent aux mathématiques scolaires dans l'école (ou plus largement, le rôle qu'ils assignent à cette discipline dont la nature est historico-culturelle et la viabilité en classe induit des interactions entre élèves, maîtres et ressources). D'autre part, l'influence des enjeux portés par les mathématiques scolaires dans la société actuelle est aussi une donnée à prendre en compte pour appréhender des aspects de cette dimension sociale chez les PE. En effet, dans le système scolaire français, les mathématiques jouent un rôle particulier dans la sélection des élèves : « dans nos sociétés, les mathématiques sont devenues un domaine fortement valorisé »(Fayol 2013, p. 3).

\section{MÉTHOdOLOGIES DE RECUEIL ET DE TRAITEMENT DES DONNÉES}

\section{Corpus}

Les neuf enseignants (PE1-PE2-PE3-PE4-PE5PE6-PE7-PE8-PE9) qui constituent notre échantillon (cf. tableau 2) ont, pour cinq d'entre eux (PE4-PE5PE6-PE7-PE8), participé au protocole de recherche sur le rapport au savoir en physique des professeurs des écoles (Decroix \& Kermen 2016).

Tous sauf PE9 disposent d'une expérience de plus de dix ans d'enseignement en cycle 3 et sept d'entre eux (tous sauf PE8 et PE9 ${ }^{3}$ ) sont actuellement en poste en cycle 3. Six d'entre eux (PE1-PE2PE3-PE4-PE6-PE8) sont maîtres formateurs; leur expertise pédagogique, reconnue par l'institution de formation, peut constituer un paramètre à prendre en compte dans nos analyses. Pour tous les PE interrogés sauf PE3 et PE5, leur projet professionnel est solidement ancré dans leur parcours d'études malgré d'éventuelles difficultés rencontrées (ruptures entre primaire et secondaire, échecs antérieurs aux concours d'enseignants). PE5, une femme, est devenue enseignante suite à une reconversion professionnelle motivée par des raisons familiales et une déception du monde de l'entreprise. PE3, un homme, a découvert le plaisir d'enseigner après un parcours d'études sinueux.

En ce qui concerne leur parcours de formation initiale, ils sont majoritairement titulaires d'un bac scientifique : sept baccalauréats scientifiques S, C, D ou E (PE1-PE2-PE3-PE5-PE7-PE8-PE9), un bac économique B (PE6) et un bac littéraire Al avec option scientifique (PE4). Les PE interrogés témoignent de cursus scolaires qui se sont déroulés agréablement à l'école primaire avec parfois des difficultés dans l'enseignement secondaire (redoublement puis réorientation après une filière $C$ pour PE8). Enfin, PE2-PE3-PE7-PE9 ont une formation initiale scientifique de niveau au moins bac +2 . Notre échantillon comprend donc un nombre significativement plus élevé de scientifiques que dans la population ordinaire des PE.

Notons encore qu'aucun d'entre eux n'a participé à des formations continues en mathématiques depuis 2008 en dehors de PE4, impliqué dans une recherche-action sur l'enseignement de la géométrie. 
Tableau 2 : Caractéristiques des professeurs des écoles interrogés 4

\begin{tabular}{|c|c|c|c|c|c|c|}
\hline $\begin{array}{l}\text { PE } \\
\text { interrogé }\end{array}$ & Sexe & Bac & Études post-bac & Ancienneté ${ }^{5}$ & $\begin{array}{l}\text { Maîtres } \\
\text { formateurs }\end{array}$ & Classe \\
\hline PE1 & $\mathrm{H}$ & $\mathrm{S}$ & Licence géographie & 12 ans & oui & $\mathrm{CM} 1 / \mathrm{CM} 2$ \\
\hline PE2 & $\mathrm{H}$ & $\mathrm{D}$ & Licence biologie & 15 ans & oui & CM2 \\
\hline PE3 & $\mathrm{H}$ & E & $\begin{array}{l}\text { DUT génie mécanique } \\
\text { Licence sciences éducation }\end{array}$ & 19 ans & oui & $\mathrm{CM} 1 / \mathrm{CM} 2$ \\
\hline PE4 & $\mathrm{F}$ & Al & $\begin{array}{l}\text { Maîtrise sciences éducation et } \\
\text { didactique des maths }\end{array}$ & 14 ans & oui & $\begin{array}{l}\text { CM2 } \\
\text { Formatrice ESPE }\end{array}$ \\
\hline PE5 & F & $\mathrm{D}$ & $\begin{array}{l}\text { DUT gestion } \\
\text { Magistère DRH }\end{array}$ & 10 ans & non & CM2 \\
\hline PE6 & $\mathrm{F}$ & B & Licence sociologie & 27 ans & oui & CE2 \\
\hline PE7 & $\mathrm{F}$ & C & $\begin{array}{l}\text { Deug MIAS } \\
\text { Licence maths physique }\end{array}$ & 12 ans & non & CM2 \\
\hline PE8 & $\mathrm{H}$ & $\mathrm{D}$ & $\begin{array}{l}\text { Licence sciences éducation } \\
\text { Maîtrise philosophie éduca- } \\
\text { tion } \\
\text { DESS ingénierie pédagogique }\end{array}$ & 20 ans & oui & Formateur ESPE \\
\hline PE9 & $\mathrm{H}$ & C & $\begin{array}{l}\text { Deug MIAS } \\
\text { Licence maths }\end{array}$ & 5 ans & non & $\begin{array}{l}\text { PLC collège mi-temps } \\
\text { Formateur ESPE }\end{array}$ \\
\hline
\end{tabular}

\section{Méthodologie de recueil des données}

La méthodologie utilisée par Charlot (1997) s'appuie sur des bilans de savoir et des entretiens. Nous n'avons pas réalisé de bilans de savoir; notre échantillon comprenant une majorité de scientifiques, ce procédé ne nous a pas semblé pertinent. L'essentiel de ce que j'ai appris jusqu'à présent dans ma vie (référence aux bilans de savoir) influe certes sur le rapport au savoir dans l'exercice du métier d'enseignant mais il nous a paru plus judicieux de tenter de l'appréhender à partir d'entretiens. Nous avons conçu des entretiens ${ }^{5}$ semi-directifs (Cappiello \& Venturini 2011) entre un chercheur et un professeur des écoles. Afin que les PE se livrent avec franchise, nous avons essayé de les traiter en professionnels. Nous n'avons donc pas posé de questions susceptibles de les déstabiliser relatives à leur maîtrise des savoirs qu'ils soient mathématiques ou didactiques.

Prenant appui sur un protocole d'entretien élaboré pour sonder le rapport au savoir en physique de professeurs des écoles (Decroix \& Kermen 2016), nous avons conçu un guide d'entretien similaire mais axé sur l'enseignement des mathématiques scolaires. Les entretiens comportent quatre étapes. Dans une première partie, nous interrogeons les PE sur leurs représentations des mathématiques en général. Dans une seconde partie, nous les questionnons sur les mathématiques qu'ils enseignent dans leur classe. Une troisième partie est consacrée aux domaines qui leur apparaissent comme sensibles (porteurs de difficultés) et aux ressources qu'ils mobilisent. Nous évoquons enfin, en quatrième partie, leur parcours de formation et les éventuelles formations en mathématiques suivies dans le cadre de leur métier.

\section{Méthodologie de traitement des données}

Les questions posées lors des entretiens ont influencé ce que nous avons recueilli sur les trois dimensions du rapport au savoir définies par Charlot. Ainsi, les analyses des enregistrements des entretiens ont donné un contenu aux dimensions et sous-dimensions du rapport au savoir mathématique scolaire des PE (que nous avions caractérisées en adaptant le cadre 
élaboré par Charlot aux enseignants). Ces définitions figurent dans les tableaux 3 et 4 .

\section{Pour la dimension épistémique}

Pour la dimension épistémique (tableau 3), nous étudions le rapport au savoir mathématique à enseigner à l'école primaire (les quatre domaines mathématiques figurant dans les programmes de 2008), le rapport à l'institution (programmes scolaires dont horaires dévolus aux différents enseignements) et le rapport à des ressources et des démarches pédagogiques et/ou didactiques.

\section{Rapport à des savoirs disciplinaires scolaires}

Les domaines de savoirs évoqués par les PE renvoient à un univers de savoirs mathématiques qui ne sont pas questionnés. Il nous a paru opportun d'identifier les traits spécifiques que les PE attribuent au savoir mathématique scolaire notamment à travers l'éventuelle hiérarchie qu'ils établissent entre les domaines mathématiques enseignés, leurs poten- tialités et leurs difficultés. La spécificité de certains domaines, de certains thèmes d'étude, les liens évoqués entre domaines mathématiques et autres disciplines sont des indicateurs que nous avons retenus.

\section{Rapport à l'institution}

La marge d'initiative plus ou moins reconnue, les contraintes des programmes, mettent en demeure les PE de témoigner ou non de la maîtrise d'un certain nombre de gestes professionnels. C'est un « Je [pris] dans la situation » (Charlot 1997, p. 81), un sujet incarné dans son statut de PE et dans son environnement qui peut évoquer son rapport à des pratiques déclarées et analysées à l'aune des programmes mais aussi de l'école. Leur interprétation personnelle des IO et la prise en compte de leurs conditions d'exercice permettent de qualifier cette sous-dimension.

\section{Rapport à des ressources et des démarches pédagogiques et/ou didactiques}

Garant du fonctionnement de l'institution « classe de mathématique », le PE a recours à de multiples ressources qui contribuent à mettre l'élève

Tableau 3 : Définitions pour la dimension épistémique

\begin{tabular}{|c|c|c|}
\hline Sous-dimensions & Définitions & Questions posées $^{7}$ \\
\hline $\begin{array}{l}\text { Rapport à } \\
\text { des savoirs disciplinaires } \\
\text { scolaires }\end{array}$ & $\begin{array}{l}\text { Présence, hiérarchie, poten- } \\
\text { tialités, difficultés des quatre } \\
\text { domaines mathématiques } \\
\text { scolaires. } \\
\text { Liens internes et externes avec } \\
\text { les autres disciplines scolaires. }\end{array}$ & $\begin{array}{l}\text { Domaines mathématiques prioritairement travaillés? } \\
\text { Partage du temps entre les } 4 \text { domaines ? Raisons? } \\
\text { Domaines qui posent davantage de difficultés? } \\
\text { Domaines les plus intéressants à travailler? Pourquoi? } \\
\text { Domaines et activités qui posent le plus de problèmes? } \\
\text { Notions pour lesquelles nous ne vous sentez pas à l'aise? } \\
\text { Pour grandeurs et mesures: } \\
\text { - Types d'activités? } \\
\text { - Liens avec autres disciplines? } \\
\text { - Types de problèmes? }\end{array}$ \\
\hline $\begin{array}{l}\text { Rapport à } \\
\text { l'institution }\end{array}$ & $\begin{array}{l}\text { Programmes scolaires. } \\
\text { Horaires dévolus aux diffé- } \\
\text { rents enseignements. }\end{array}$ & $\begin{array}{l}\text { Comment tirez-vous parti du BO en cycle 3 ? } \\
\text { Partage du temps entre les } 4 \text { domaines? Raisons? Impact } \\
\text { sur vos pratiques des évolutions des programmes? } \\
\text { Domaines les plus intéressants à travailler? Pourquoi? } \\
\text { Domaines qui posent le plus de problèmes? } \\
\text { Nature des difficultés rencontrées? } \\
\text { Notions pour lesquelles nous ne vous sentez pas à l'aise? } \\
\text { Pour grandeurs et mesures : } \\
\quad \text { - Types d'activités? } \\
\quad \text { - Liens avec autres disciplines? } \\
\quad \text { - Types de problèmes? } \\
\text { Pourquoi faut-il faire des maths à l'école primaire? Liens } \\
\text { avec d'autres disciplines? }\end{array}$ \\
\hline
\end{tabular}




\begin{tabular}{|c|c|c|}
\hline $\begin{array}{l}\text { Rapport à } \\
\text { des ressources et des } \\
\text { démarches pédagogiques } \\
\text { et/ou didactiques }\end{array}$ & $\begin{array}{l}\text { Ressources matérielles. } \\
\text { Démarche de résolution de } \\
\text { problèmes. } \\
\text { Intérêt du recours à la mani- } \\
\text { pulation. }\end{array}$ & $\begin{array}{l}\text { Ressources sur lesquelles vous vous appuyez? } \\
\text { Manuels de référence? } \\
\text { Types d'activités évoqués par le mot « mathématiques »? } \\
\text { Types d'activités les plus intéressants à travailler? } \\
\text { Domaines et activités qui posent le plus de problèmes? } \\
\text { Séance réussie ? Et moins réussie? } \\
\text { Comment introduisez-vous une nouvelle notion? } \\
\text { Pour grandeurs et mesures: } \\
\quad \text { - Types d'activités? } \\
\quad \text { - Liens avec autres disciplines? } \\
\quad \text { - Types de problèmes? }\end{array}$ \\
\hline
\end{tabular}

en activité (en capacité de construire un rapport au savoir tel que le PE l'envisage). Nous entendons par ressources de l'enseignant tout ce dont il peut faire usage dans sa pratique pour créer les conditions favorables à la mise en activité des élèves en amont comme en action. La présence ou l'absence de diverses ressources (manuels, sites internet), de types d'activités (dont la résolution de problèmes et le recours à la manipulation) peut éclairer le rapport au savoir du $\mathrm{PE}^{6}$.

\section{Pour la dimension identitaire}

Pour la dimension identitaire (tableau 4), nous recherchons le sens et la valeur que représente l'enseignement des mathématiques scolaires pour les PE en termes de rapport à soi (rapport personnel aux mathématiques, mobilisation à enseigner les mathématiques scolaires, rapport à la formation initiale et continue) mais aussi de rapport aux autres (inspecteurs, collègues, élèves).

\section{Rapport à soi}

Cette sous-dimension recouvre tout ce qui fait référence à l'image de soi du PE à travers « l'apprendre ", quelles que soient les figures sous lesquelles il se présente « objets, activités, dispositifs et formes » (Charlot 1997, p. 77) mais aussi sous lesquelles il s'est présenté dans la trajectoire identitaire du sujet. La notion de légitimité (par rapport à des compétences personnelles) qui renvoie sans conteste à une dimension sociale est appréhendée à travers l'expression d'éléments de discours qui évoquent le bien vivre du PE ou a contrario ses difficultés. La capacité à évoquer un rapport à des mathématiques rencontrées dans la trajectoire de formation et à lier ce rapport et le rapport actuel aux mathéma- tiques à enseigner est encore un moyen de spécifier le rapport à soi.

\section{Rapport aux autres}

Le rapport aux autres du PE s'exprime par le biais des échanges, des interactions, avec les élèves d'abord, mais aussi avec l'ensemble des acteurs du système éducatif (supérieurs hiérarchiques, collègues). Il est caractérisé en termes d'impact sur des pratiques déclarées (ce que les PE disent de leurs activités en acte), le poids de certaines interactions pouvant s'avérer différenciateur. De même qu'en termes de rapport à soi, le rapport aux autres renvoie par nature à la dimension sociale du rapport au savoir du PE.

\section{Pour la dimension sociale}

La dimension sociale s'est révélée délicate à démêler car imbriquée dans les dimensions épistémique et identitaire. La transversalité de la dimension sociale nous a conduites à la caractériser à partir des déclarations des $\mathrm{PE}$ sur les différentes questions ${ }^{8}$ relevant des dimensions épistémique et identitaire.

\section{ANALYSE DES DONNÉES}

Cette étude exploratoire s'appuie sur une analyse qualitative des éléments de discours des PE. Elle nous a permis de dégager des tendances plus ou moins singulières quant à l'influence du rapport au savoir mathématique des PE sur leurs pratiques déclarées. Ainsi celles-ci peuvent être liées de façon différenciée à des dimensions du rapport au savoir. Nous avons choisi de catégoriser les traces de ces pratiques à partir du recueil de nos entretiens. 
Tableau 4 : Définitions pour la dimension identitaire

\begin{tabular}{|c|c|c|}
\hline Sous-dimensions & Définitions & Questions posées \\
\hline $\begin{array}{l}\text { Rapport } \\
\text { à soi }\end{array}$ & $\begin{array}{l}\text { Légitimité ou non à enseigner } \\
\text { les maths. } \\
\text { Formation initiale et continue. }\end{array}$ & $\begin{array}{l}\text { Que représentent les maths pour vous? Souvenir de votre première } \\
\text { rencontre avec les maths dans votre enfance? } \\
\text { Avez-vous le sentiment de faire ou utiliser des maths dans votre vie } \\
\text { de tous les jours? } \\
\text { Formation universitaire d'origine? } \\
\text { Activité professionnelle avant d'être PE? } \\
\text { Formation reçue en maths? } \\
\text { Formation continue, animations pédagogiques en maths? } \\
\text { Y -a-t-il des notions pour lesquelles vous ne vous sentez pas à } \\
\text { l'aise? } \\
\text { Difficultés rencontrées le plus souvent et dispositifs mobilisés pour } \\
\text { les résoudre? } \\
\text { Avez-vous peur d'éventuelles questions des élèves? }\end{array}$ \\
\hline $\begin{array}{l}\text { Rapport } \\
\text { aux autres }\end{array}$ & 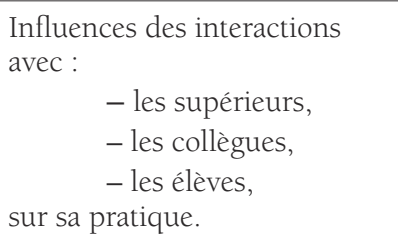 & $\begin{array}{l}\text { Pratiquez-vous le travail en équipe? } \\
\text { Types d'activités les plus intéressants à travailler ? Pourquoi ? } \\
\text { Difficultés rencontrées le plus souvent et dispositifs mobilisés } \\
\text { pour les résoudre? } \\
\text { Avez-vous peur d'éventuelles questions des élèves? }\end{array}$ \\
\hline
\end{tabular}

\section{Dimension épistémique}

\section{Rapport à des savoirs disciplinaires scolaires}

Lors des entretiens, les PE interrogés n'évoquent pas des défauts de connaissances pour les savoirs disciplinaires enseignés à l'école primaire.

La répartition des enseignements entre les quatre domaines ${ }^{9}$ figurant dans les Instructions Officielles de 2008 en vigueur au moment de notre étude n'est appliquée à la lettre de manière hebdomadaire que par PE2-PE7. Ainsi, PE3-PE4-PE8-PE9 tendent à établir un équilibre global au moyen d'une progression spiralaire (PE4-PE8-PE9) ou d'un module qui se déroule sur trois semaines puis donne lieu à un rééquilibrage (PE3).

Six d'entre eux (PE1-PE3-PE4-PE5-PE6-PE8) accordent une importance prédominante au domaine «Nombre et calcul » pour lequel ils mettent en place un travail dans la durée. PE6-PE8 citent explicitement les enjeux de la numération tandis que PE3 évoque ceux du calcul : « Le calcul prioritairement parce que j'estime, on disait, le principal, c'est que les élèves sachent lire et calculer [...] dans ces grandes phrases, on peut quand même prendre une part de vérité, c'est vrai, le calcul, c'est important [...], le rapport au calcul constitue la base ». Par contre, PE5 considère les « grands nombres » comme peu pertinents et PE2 souligne que le travail sur le champ numérique au cycle 3 est plus réducteur que le travail dans le domaine de la gestion de données. Le calcul mental est cité deux fois : activité régulière faisant l'objet de débats en classe pour PE2, travail de fond sur les tables d'addition et de multiplication pour PE5. La géométrie, bien que minorée, est plébiscitée pour ses aspects concrets (PE1-PE4), instrumentaux (PE5), en lien avec les arts (PE5). Seul PE9 indique apprécier les grandeurs et mesures ainsi que la gestion de données qui sont des domaines qu'il juge moins chronophages. PE9 souligne de plus que les grandeurs et mesures permettent de donner du sens aux activités de résolution de problèmes. Enfin, la gestion de données est également le domaine mathématique préféré de PE2 (qui apprécie les liens que l'on peut faire, par exemple, avec les techniques opératoires) et de PE8 (pour qui elle présente l'intérêt de favoriser des synthèses sur différents éléments de maths du cycle 3).

Partiellement déchargés de leur classe en raison de leur statut de maîtres formateurs, PE3-PE4-PE8 prennent cependant en charge tous les domaines mathématiques tandis que PE6 choisit de déléguer la géométrie et PE1-PE2 les grandeurs et mesures. 
Certains thèmes d'étude sont jugés délicats à enseigner au cycle 3. Tout d'abord les grandeurs et mesures sont pointées par cinq PE (PE1-PE5-PE7PE8-PE9). Pour PE7, « Il y a un problème d'abstraction avec les élèves pour le domaine des grandeurs et mesures; je me demande à quel âge est fait le lien entre numération et mesure. Il faut manipuler, faire le lien avec la vie de tous les jours...». Les réticences de PE5 par rapport aux aires et aux volumes s'expriment en termes de difficultés pour les élèves à apprendre et utiliser des formules : « Je ne vois pas l'intérêt de formaliser l'aire d'un carré, paver, compter oui. Pourquoi la formule?». Ensuite, PE2-PE4PE5-PE9 rapportent des difficultés avec les fractions et les décimaux (problèmes de représentation de nouveaux nombres). Pour PE9, « Il faut y passer du temps ». Enfin, PE4 cite des difficultés avec la proportionnalité (tableaux de proportionnalité) et PE6 avec la numération (extraction du nombre de dizaines dans un grand nombre).

Quelques enseignants envisagent les mathématiques comme un outil au service des autres disciplines scolaires et réciproquement les apports éventuels des autres disciplines pour apprendre les mathématiques. Le domaine organisation et gestion de données, emblématique de ce point de vue, est mis en relation avec d'autres disciplines : avec la géographie (ombres thermiques pour PE7, le calcul de densité de population pour PE2 et la lecture d'une pyramide des âges pour PE9). PE5 indique tisser des liens entre la géométrie et l'art (le graphisme des années soixante-dix et l'œuvre de Mondrian) ainsi que l'EPS car « La géométrie, cela va bien avec les Arts Plastiques et l'EPS. Quand on est bien dans l'espace, bien dans son corps, on a confiance en soi, on arrive à se positionner dans le groupe ». Des liens entre les mathématiques et le français, plus particulièrement entre lecture et résolution de problèmes, sont aussi soulignés par le biais des difficultés rencontrées en résolution de problèmes (PE4-PE5). Certains d'entre eux évoquent les liens entre les domaines mathématiques : le calcul outille le travail dans tous les autres domaines (PE3), les grandeurs et les mesures donnent sens à la numération (PE2), les liens entre géométrie et grandeurs et mesures sont intéressants (PE4). Pour PE5, résolution de problèmes et calcul mental sont complémentaires: "Je fais une fixation : décomplexer les élèves sur les tables; je donne deux ou trois nombres dans une table. Qu'est-ce qu'on peut raconter? Un énoncé de problèmes... ».
Les enjeux de savoir sont présents, comme en témoigne la constellation de notions citées dans les activités privilégiées, mais les tâches "manipuler, découper, calculer, se poser des questions, comparer, schématiser, résoudre des problèmes $»$ (PE1-PE4PE5-PE6-PE7-PE8) restent circonscrites à des thèmes d'étude mathématiques. Faire des maths à l'école, c'est pour « Apprendre à raisonner, se poser des questions, acquérir des outils...» (PE5), « Devenir autonome » (PE8), « Avoir les bases pour le secondaire » (PE9), « Avoir un bon métier » (PE5) et les finalités, bien que de natures diverses selon les PE interrogés, privilégient une dimension transversale (se poser des questions, apprendre à raisonner).

\section{Rapport à l'institution}

Les enseignants interrogés connaissent et suivent les Instructions Officielles en vigueur. Chez ces PE chevronnés, les contraintes institutionnelles (conformité à des injonctions officielles, contrôle de l'inspection, travail d'équipe) ne sont pas vraiment questionnées.

Seuls PE4-PE5 remettent en question la pertinence de certaines notions des programmes pour lesquelles le niveau d'exigence leur semble trop élevé, sans rejet explicite : grandeurs et formules jugées inaccessibles pour PE5, tableau de proportionnalité et problèmes complexes trop compliqués pour PE4, fractions vues trop tôt « Je ne comprends pas pourquoi les fractions sont au programme de l'école élémentaire. Il y a un problème de maturité des enfants » pour PE5.

PE5 reconnaît que "les gens qui font les programmes donnent le "la" » et que les programmes permettent des prises de conscience : "j'avais tendance à négliger la gestion de données ». Mais les évolutions que pourraient entraîner les changements de programmes ne se traduisent qu'en termes de démarches pédagogiques : "À chaque fois, je me débrouille pour savoir si ce que je fais est conforme avec la demande institutionnelle [...] à force d'apprentissage, je modifie un peu les dispositifs d'apprentissage et les entrées didactiques, les processus, non $»(\mathrm{PE} 3)$.

PE9 signale toutefois les difficultés temporelles que peut soulever la lecture du programme : «Si on veut passer du temps sur chaque notion, on va peutêtre avoir l'impression d'un grand manque de temps 
avec les programmes $[\ldots]$; le défaut des programmes, c'est peut-être celui-ci : si on n'arrive pas à construire des activités imbriquées les unes dans les autres, les activités les unes à côté des autres vont nous donner l'impression d'un manque de temps, on va courir après le temps à partir de la période cinq ».

Il semble que les Instructions Officielles de 2008 en vigueur au moment de notre étude ne suscitent un questionnement concernant certains domaines et thèmes mathématiques (la gestion de données, la proportionnalité et la règle de trois, les grandeurs et mesures, les formules d'aires, les conversions) que pour très peu d'entre eux (PE4-PE5) et ce questionnement renvoie alors à des arguments tels que «Les élèves sont trop petits » (PE5). Si les liens avec d'autres enseignements (la géographie, l'art plastique, l'EPS), le français (la maîtrise de la lecture) sont évoqués, ils ne signalent pas une prise en compte nouvelle des injonctions officielles.

\section{Rapport à des ressources et des démarches pédagogiques et/ou didactiques}

\section{Les ressources}

Les PE utilisent des manuels diversifiés. Pour PE8, « Il me fallait absolument un manuel de référence, plus le guide du maître, pour donner les cautions scientifiques $»$. PE3-PE4-PE5-PE7-PE9 font référence à la collection ERMEL $(2000,2006)$. Ils font encore usage de manuels (Cap maths pour PE1-PE4-PE5-PE7-PE9) ou de ressources internet (Sésamath pour PE4-PE7 ou le site personnel d'un enseignant pour PE5) qui témoignent d'une curiosité ciblée sur l'enseignement des mathématiques.

Ils utilisent ces ressources en les adaptant à leurs élèves : guides rassurants pour organiser et finir le programme dans le cadre d'un cours double (PE8); choisies avec d'autres et parmi d'autres mais avec recul pour leur utilisation de la démarche expérimentale (PE7); sources d'inspiration mais réadaptées en raison d'une classe hétérogène (PE4). Comme le signale PE9 « Je me suis beaucoup servi des ERMEL pour construire les activités, les situations problèmes et puis sinon j'inventais, j'essayais d'inventer ce que je faisais et surtout j'avais une énorme ressource : des vieux instits autour de moi, dans la famille [...]; ERMEL reste une référence mais c'est pas un manuel pour les élèves [...] J'ai jamais cru en la vérité d'un manuel, s'il y avait un manuel meilleur que les autres, tout le monde achèterait celui-ci et on ne s'embêterait plus ».

\section{Le recours à la manipulation}

Le recours à la manipulation, marqueur fort de l'activité mathématique à l'école, est plébiscité par presque tous les PE interrogés. PE6 cite Confucius : « J'entends, j'oublie. Je vois, je me souviens. Je fais, je comprends » et ajoute que la manipulation est indiquée même pour des élèves de cycle 3 . Il faut donc : « Manipuler, comparer, schématiser, résoudre, compter, abstraire » (PE8). Pour PE1, la manipulation permet de différencier les apprentissages. Pour PE4-PE6, la manipulation est justifiée par la nature abstraite des concepts mathématiques. Par exemple, « Les élèves manquent de représentations de ce qu'est un nombre $»$ (PE6).

Mais peu de PE interrogés citent des situations explicites où l'intérêt du recours à la manipulation est manifeste. Par exemple, PE4 tire profit de la manipulation avec ses élèves de cycle 3 pour faciliter l'apprentissage des nombres rationnels et cite les bandes à plier et découper pour introduire les fractions. PE9 évoque une séance de géométrie particulièrement satisfaisante où par découpage, collage, coloriage, les élèves ont découvert les propriétés des quadrilatères. PE5, peu favorable à l'introduction des formules, légitime cependant la notion de périmètre, car « On est dans le concret, on utilise une ficelle».

Ces enseignants interrogés semblent donc plébisciter un schéma prédominant : manipuler puis abstraire, faire pour comprendre. Citons encore PE4 : «Mes activités privilégiées : les démarches d'expérimentations, les manipulations ; j'essaie de faire manipuler le plus possible pour arriver à l'abstraction ».

\section{La démarche de résolution de problèmes}

Parmi les activités privilégiées, beaucoup évoquent le rôle majeur des situations-problèmes. Nous retrouvons de multiples références aux situationsproblèmes pour introduire des notions (PE1-PE6) et à la résolution de problèmes pour utiliser des outils (PE5-PE8-PE9) mais peu de références à une typologie explicite des problèmes et de leurs fonctions.

Pour PE9, comme pour plusieurs PE, « il est important de travailler sur le sens des notions et donc de faire de la résolution de problèmes ». PE6 insiste sur la démarche et ses difficultés : « Le choix des situations problèmes est crucial pour introduire de nouvelles notions. Il est important de montrer 
les phases de recherche et de réaliser les mises en commun même sur les démarches fausses. Ne pas gommer, rayer, pour montrer la démarche de recherche ». PE4 souligne les difficultés de mise en œuvre: "Les problèmes de recherche et les problèmes complexes ne font pas sens. Les élèves ne trouvent pas les étapes, s'interdisent de schématiser de faire un brouillon; ils ne mettent pas de sens, veulent donner tout de suite la réponse; c'est le même problème avec la démarche d'investigation - ils ne cherchent pas, veulent la réponse très vite » et insiste sur le rôle d'un travail sur la lecture, la compréhension, l'interprétation du problème.

PE6 oppose l'apprentissage d'une démarche et l'application d'une technique. «Il est important de passer du temps sur le sens et la compréhension. Je suis indisposé par les tableaux de numération. Les tableaux représentent des outils qui peuvent permettre de gagner du temps mais ils doivent être présentés aux élèves une fois que le sens est installé et construit avec les élèves. »

Les problèmes qui donnent du sens aux apprentissages sont pour PE2 des situations qui relèvent de la vie quotidienne: "Je leur tends des perches, je propose des situations quotidiennes. Faire des courses, mais ils n'en font plus! Avec la carte bancaire, ils n'ont plus d'argent de poche. Je prends des exemples utiles quand je bricole... J'utilise des énoncés pratiques (qu'ils vivront plus tard) » mais il exprime aussi un certain désabusement. PE5 plébiscite également le recours à des problèmes très concrets, issus de situations quotidiennes et met l'accent sur les apprentissages techniques : « Donner des repères, des outils pour qu'ils se sentent bien, plutôt que les noyer. Ils sont petits; on en perd, on en perd » et insiste sur le « conditionnement » et la «systématisation» qui lui paraissent indispensables. PE8 apprécie les problèmes qui permettent aux élèves d'utiliser les quatre techniques opératoires.

Certaines phases de l'activité de résolution de problèmes, telle la trace écrite, sont perçues comme plus délicates. Ainsi PE4 qui plébiscite les démarches d'expérimentations relève : "J'essaie de faire manipuler le plus possible pour arriver à l'abstraction; je fais les traces écrites avec eux mais cela prend la moitié du temps : je différencie pour certains (mots-clés au tableau, texte à trous) ».

Notons encore que si les apprentissages numériques (le calcul, les techniques opératoires) sont souvent liés à la résolution de problèmes, c'est rarement le cas pour la géométrie (PE9).

\section{Dimension identitaire}

\section{Rapport à soi}

Nous avons recueilli peu de traces sur comment ils ont appris les mathématiques et celles-ci prennent davantage la forme d'éléments sur comment ils n'ont pas pu les apprendre. Pour PE1-PE4-PE5-PE6-PE8, les mathématiques ont été source de mal-être scolaire (en primaire pour PE5; au collège pour PE4-PE6 ou au lycée pour PE1-PE8). Plus précisément, PE8 est resté en grande souffrance morale tandis que PE1-PE4-PE5-PE6 ont retrouvé depuis confiance en leurs capacités avec la discipline. Près de la moitié d'entre eux témoigne d'une appétence explicite et fait état d'un intérêt (PE2-PE7-PE9) ou même d'une fascination (PE3-PE5) pour ce domaine qui échappe au scolaire (dont PE5 dont nous avons souligné les rapports difficiles avec les mathématiques durant sa scolarité).

Comme les PE interrogés ont presque tous (sauf PE9) plus de dix ans d'ancienneté, nous pouvons nous interroger sur l'impact éventuel de la formation qu'ils ont reçue dans les Écoles Normales (PE6-PE8) et en IUFM (PE1-PE2-PE3-PE4-PE5-PE7-PE9). Tous les enseignants interrogés considèrent maîtriser l'aspect théorique des notions à enseigner (aspect perçu comme dominant dans la formation reçue dans les IUFM).

PE4-PE9 font explicitement référence à des connaissances professionnelles issues de la formation. Seul PE9, avec une formation initiale scientifique en mathématiques, reconnaît spontanément que la formation a répondu à ses besoins en pédagogie et en didactique : «Moi, j'ai eu la chance de faire deux fois l'IUFM, en tant que PE, puis en tant que PLC; j'ai trouvé des choses très, très bien; j'ai rencontré des gens formidables... Même, si ça n'était pas des formations, c'était des discussions avec des gens très bien. [...] Moi, j'avais quand même besoin de la formation didactique, pédagogique en mathématiques. À l'époque, on ne sait rien, on sort de licence, on sait faire des maths, heureusement qu'il y avait la formation derrière ». PE4, après un questionnement plus précis, admet avoir reçu en deuxième année de formation des apports didactiques perti- 
nents (dans une section spéciale, après avoir été admis sur liste complémentaire puis envoyé sur le terrain pendant un an): « Si, si, si je dis n'importe quoi, si j'ai eu de la didactique en PE2 et c'était super, j'ai eu Mme... ». Au contraire, PE3 considère avoir appris peu de choses à l'IUFM en maths : " Je me suis fait moi-même ».

La référence à des apports didactiques est plus ou moins tangible dans la description des activités privilégiées par les $\mathrm{PE}$ avec parfois une focalisation sur certaines difficultés (l'institutionnalisation pour PE4). Pourtant, les références aux ressources ERMEL (2000, 2006) (PE3-PE4-PE5-PE9) peuvent traduire un certain impact de la formation initiale car il s'agit de manuels pédagogiques de référence en formation des PE.

Notons que PE4, seul enseignant interrogé ayant participé à une recherche-action (en géométrie), a vu son opinion sur ce domaine mathématique changer radicalement : " Avant, j'avais horreur de la géométrie ». Elle indique maintenant préférer enseigner la géométrie en raison de son aspect ludique.

\section{Rapport aux autres}

\section{Inspecteurs}

Si les enseignants interrogés suivent les Instructions Officielles en vigueur, aucun d'entre eux n'évoque ses rapports avec l'Inspection.

\section{Collègues}

Les PE interrogés font état d'un travail collaboratif très fluctuant qu'ils souhaiteraient plus intense. Les enseignants citent des collaborations allant d'un véritable travail de mutualisation (PE7), à l'organisation de rallyes et de challenges (PE3), en évoquant la nécessité d'aider un collègue qui « débarque » (PE5) car « la charge de travail actuelle est trop lourde ». PE5 ajoute : "J'ai beaucoup appris au contact de mes collègues ». PE8 déplore que ce travail collaboratif soit souvent « fait en amont mais pas en aval» tandis que PE4 souligne l'inertie d'une équipe « momifiée dans ses routines individuelles ». Par contre, sans incitation formelle, PE3 offre sa classe ou ses activités (géométriques notamment) au regard de ses collègues: «Vois ce que je fais, si cela t’intéresse ».

Cependant, les PE concernés sont pour la plupart maîtres formateurs et assurent un statut et des charges de travail qui peuvent les marginaliser. PE2 parle de « bonnes intentions» associées à un « gros manque de temps » car « les sollicitations de l'ESPE sont jugées trop lourdes ». Pour PE3, « les maîtres formateurs ne se voient que très peu »dans les écoles d'application où ils sont déchargés de leurs classes sur des jours différents dans la semaine. Notons encore la spécificité des conditions de travail de PE9 qui, titulaire mobile, n'a pas pu s'investir et bénéficier d'un travail d'équipe.

\section{Élèves}

Les enseignants que nous avons interrogés se sentent tous légitimes dans leurs fonctions vis-à-vis des élèves dont ils ne craignent pas les questions. Deux PE l'explicitent : PE8 évoque son expérience, PE3 accepte les questions et se renseigne, PE3 s'appuie sur l'aide éventuelle des élèves (pour comprendre certaines difficultés des élèves, PE3 désigne d'abord un élève tuteur qui va expliquer à l'élève en échec, puis exploite l'explication).

Leur interprétation des besoins, des attentes, du ressenti des élèves, joue un rôle important sur leurs pratiques déclarées : tandis que certains (PE3-PE9) décrivent les activités qu'ils privilégient en termes de thèmes de savoir et de sujet d'études, d'autres lient précisément ces activités à la mobilisation des élèves (le calcul mental pour PE2), à leur motivation par des jeux ou des petits problèmes concrets (PE5). Pour PE5, la prise en compte des besoins des élèves est prioritaire : « Il y a un fossé que l'on creuse si l'on ne tient pas compte de la réalité des enfants».

Par contre, leur attitude face aux difficultés des élèves diffère. Pour PE1, le niveau d'exigence doit absolument être maintenu : "Même face à des élèves en difficulté, il ne faut pas baisser le niveau d'exigence ». Mais, pour d'autres (PE2-PE4-PE5), le niveau d'exigence est difficile à maintenir car « Les élèves manquent d'attention, ils zappent et il est important de fractionner les apprentissages » (PE2); « les élèves sont incapables d'aller au bout d'une résolution de problème » (PE4); «C'est compliqué [...], Les élèves sont trop petits [...], C'est pour alléger $[\ldots]$ », « Les difficultés de compréhension des élèves sont de plus en plus grandes », " Il faut faire le deuil de vos attentes par rapport à ce que l'on réussit avec les élèves » (PE5). D'autres s'adaptent et dédramatisent : " En maths, il n'y a pas de problèmes mais des solutions " $[\ldots]$ « Je ne fais pas des maths avec mes élèves mais des maths et moustiques. Parfois, 
cela pique, mais il y a toujours une solution pour calmer la douleur $»$ (PE6).

\section{Dimension sociale}

Les PE interrogés sont convaincus de l'utilité des mathématiques, à l'école et en dehors de l'école. Les mathématiques représentent un mode de pensée et un outil interdisciplinaire et culturel. Les enseigner étaye par ailleurs le sens et les valeurs véhiculés par leur fonction sociale.

Tous les PE reconnaissent donc la légitimité de l'enseignement des mathématiques à l'école. Elles constituent un outil pour maîtriser les pratiques de la vie quotidienne. Les mathématiques permettent d'accéder à des savoirs scolaires par des activités telles que «manipuler, découper, schématiser, calculer, compter...» dont nous avons souligné les implicites en termes de compétences transversales. Nombre d'entre eux les conçoivent aussi comme un outil au service des autres disciplines. Pour PE6-PE7PE8-PE9, les mathématiques du primaire représentent une première approche avec une démarche scientifique : "Il faut faire des maths à l'école primaire pour pouvoir s'interroger sur un objet, faire discuter les élèves, schématiser un problème et le résoudre, pour maîtriser davantage son autonomie » (PE8). Tous citent ensuite l'utilité des mathématiques dans le quotidien et l'utilité à vivre dans le monde (les statistiques, les jeux pour PE4).

Certains regrettent le rôle sélectif joué par les mathématiques dans l'orientation scolaire des élèves. Car les mathématiques portent aussi un enjeu social à plus long terme pour le futur individu dans la société (PE4-PE5) et pour le futur élève du secondaire (PE6-PE8-PE9). PE6 nous livre : « Les élèves éprouvent de l'appréhension vis-à-vis des maths car, discipline reine, les maths représentent un moyen de sélection. Cela génère des angoisses. La société est très scientifique et cela constitue une pression pour les élèves. Cela est d'autant plus marqué que l'on avance dans le cursus, surtout en études supérieures. Il faut présenter les maths comme un jeu et dépasser les représentations des maths comme une discipline élitiste pour obtenir un peu de décontraction ». PE8 s'estime "meurtri par le système de sélection vécu » qui l'a « orienté par les maths » vers un bac scientifique (d'abord un bac E auquel l'enseignant a échoué puis un bac D obtenu) alors qu'il aurait souhaité faire un bac économique (B) et que cette trajectoire l'a conduit à « un dégoût absolu des maths » sans pour autant obérer son plaisir à enseigner les mathématiques à l'école.

Ces éléments, selon notre cadre, éclairent la dimension sociale d'un certain rapport au savoir mathématique scolaire. Ils ne peuvent pas spécifier le rapport à l'enseigner des PE marqué par leur appartenance sociale au monde éducatif et leur polyvalence mais seulement une facette de ce rapport : leur conception du rapport au savoir mathématique envisagé pour leurs élèves.

\section{RÉSULTATS}

Nous sommes conscientes des limites de notre recherche exploratoire qui porte sur un nombre très réduit d'enseignants non représentatifs de la communauté des PE. Le modèle du rapport au savoir développé par Charlot (1997) nous a toutefois permis de mieux comprendre comment les professeurs des écoles interrogés assument leur rôle d'enseignant polyvalent en charge d'enseigner les mathématiques à leurs élèves. La mobilisation des PE pour enseigner les mathématiques résulte de l'intrication des dimensions identitaire et épistémique d'un rapport au savoir issu de leur trajectoire personnelle subordonné à une dimension sociale (liée à leur statut de PE chevronné et à leur perception du rôle des mathématiques dans la société).

Première question de recherche : peut-on trouver des spécificités du rapport au savoir mathématique scolaire de professeurs des écoles expérimentés?

Nous retenons trois spécificités du rapport au savoir mathématique scolaire des professeurs des écoles. Tout d'abord, les PE interrogés cherchent à motiver les élèves pour qu'ils se mobilisent dans les activités mathématiques. Ensuite, les PE visent le développement de compétences transversales mais aussi l'appropriation des bases fondamentales en calcul (utilité dans la vie quotidienne). Enfin, leur rapport aux mathématiques scolaires (qui peut légitimer le statut social du PE) présente dans leurs propos tous les traits d'une conformité aux exigences 
institutionnelles éducatives et politiques (programme et socle commun).

\section{Première spécificité : les PE interrogés cherchent à motiver les élèves pour qu'ils se mobilisent dans les activités mathématiques.}

\section{D’un point de vue épistémique}

Les PE interrogés font référence à une diversité de ressources allant de sites internet à des manuels de référence pour les élèves, des guides pratiques permettant un usage « clé en main » pour le maître mais aussi à des ouvrages tels que ceux de la collection ERMEL $(2000,2006)$. Cette collection constitue une interface entre les apports de la recherche en didactique et les pratiques de classe. Les besoins d'une recherche documentaire qui évolue au cours du temps, la nécessité d'adapter, voire de puiser dans les expériences d'autres enseignants, montrent que les PE cherchent à concilier les enjeux de savoirs présentés dans les programmes et la création de ressources adaptées pour les élèves.

" Manipuler pour parvenir à l'abstraction», « Faire, passer par le vécu pour apprendre » (PE4, PE6, PE8, PE9) et aussi, mais un peu moins fréquemment « se questionner » (PE8) sont des principes souvent évoqués pour caractériser la démarche d'apprentissage. Une certaine focalisation des PE sur la manipulation met en évidence l'importance accordée à la mise en activité de l'élève. Nous n'écartons pas les enjeux des savoirs à construire mais la démarche plébiscitée met en exergue le développement de compétences transversales sans référence à des objets d'étude explicites (nous y reviendrons dans le paragraphe suivant à propos de la deuxième spécificité dégagée). Le lien éventuel qui pourrait être fait entre manipulation et résolution de problèmes (la manipulation comme moyen d'entrer dans le problème, d'émettre des hypothèses, de valider) est parfois évoqué.

L'influence des programmes marque les propos des PE. Face aux difficultés constatées chez les élèves, les manuels scolaires ont glissé dans les années 1990 vers une approche méthodologique consistant à analyser des énoncés, trier des informations utiles ou inutiles, sans que cela apporte une aide réelle à la compréhension du problème (Houdement 2003). Cette approche méthodologique relative au traitement de l'information, inspirée des sciences cognitives, semble encore d'actualité chez certains PE. Elle peut renforcer la volonté des PE d'articuler travail sur la lecture et résolution de problèmes.

Tous les PE interrogés s'appuient sur la résolution de problèmes. Mais la démarche de résolution de problèmes qui constitue en filigrane la démarche d'apprentissage des mathématiques, garante du sens des savoirs, est adaptée au contexte des classes. Les PE mettent en œuvre, d'une part des petits problèmes concrets pour le calcul, le sens, d'autre part des «word-problem » avec énoncé complexe, voire problèmes ouverts (pour chercher) dont la mise en œuvre compte tenu des difficultés des élèves est difficile à gérer.

\section{D’un point de vue identitaire}

Si apprendre des mathématiques dans le passé a été source de mal-être pour certains $\mathrm{PE}$, le rapport à l'apprendre que les PE ont construit en tant qu'enseignant est apaisé. Parce qu'il prend en compte le développement des compétences transversales de l'élève et mobilise chez le PE des savoirs qui ne sont pas purement disciplinaires, le rapport à l'apprendre à l'école primaire peut permettre que s'instaure un nouveau rapport aux mathématiques scolaires.

Plus encore, l'attirance disciplinaire (Develay 1995) vers les mathématiques n'est pas forcément un outil pour mieux transmettre. L'un des PE, de formation scientifique, peine à entrer dans le cheminement cognitif de l'élève qui ne comprend pas. Au contraire, l'attirance disciplinaire vers des domaines non mathématiques peut permettre de mieux comprendre les difficultés des élèves. Ainsi, un autre PE au profil littéraire, se focalise sur la démarche compréhensive de la notion avant sa caractérisation en termes d'outil.

L'impact de la formation initiale dans les IUFM sur le rapport au savoir en mathématiques des PE ne semble pas sans lien avec des constats exprimés par Peltier (1995). La formation peut permettre une remise à niveau et une certaine réconciliation avec les mathématiques. Elle permet aussi une prise de conscience : le corps des savoirs pour enseigner n'est pas le corpus des connaissances de base. Et elle met aussi en évidence une évolution dans les conceptions déclarées des professeurs débutants (stagiaires) sur l'enseignement et l'apprentissage des élèves due à l'influence d'un modèle socio-constructiviste.

Les autres, dans les propos que nous avons relevés, ce sont essentiellement les élèves. Tous les PE interrogés cherchent prioritairement à enrôler les élèves pour apprendre les mathématiques. 
En lien avec la dimension sociale

Les propos retenus sur l'utilité des mathématiques, la légitimité de les enseigner et la nécessité pour certains d'entre eux d'éluder leur caractère élitiste, permettent de préciser le sens et la valeur que les PE octroient au savoir mathématique à enseigner aux élèves. Dimension sociale, parce que les mathématiques scolaires constituent un bagage minimal pour devenir citoyen autonome, parce que réconcilier les élèves avec les mathématiques, c'est reconnaître la pertinence d'un domaine de savoir incontournable, parce que transmettre des savoirs mathématiques légitime leur posture de PE.

\section{Deuxième spécificité : les PE visent le} développement de compétences transversales mais aussi l'appropriation des bases fondamentales en calcul (utilité dans la vie quotidienne)

D’un point de vue épistémique, les PE interrogés accordent une attention délibérée au domaine numérique. La stabilité de ce domaine, peu sensible aux réformes, permet que le rapport au savoir du PE en posture d'enseignant soit naturalisé (expérience), assez peu éloigné du rapport qu'il entretenait en posture d'élève. De même, pour certains (non formés et peu à l'aise avec ce domaine), la géométrie préserve le statut de science déductive, sélective (source de malaise) qu'elle représentait en posture d'élève.

Toutefois, lors des entretiens, les PE n'ont pas clairement marqué la différence entre, d'une part, l'étude du concept de nombre et de ses extensions successives et, d'autre part, l'étude de la numération et des différentes désignations des nombres.

Parmi les domaines ou thèmes relevés comme délicats à enseigner, il faut distinguer la nature de ces difficultés. Les difficultés identifiées par les PE pour les fractions et la proportionnalité sont inhérentes à ces apprentissages et identifiées en didactique. Quelle que soit la théorie sous-jacente (théorie des grandeurs ou numérique), il s'agit d'un saut conceptuel pour les élèves (nouveaux nombres, nouvelle relation entre grandeurs, nombres et opérations). Le niveau de conceptualisation de ces notions sous-tend un difficile apprentissage dans la durée, initié seulement en cycle 3. Par contre, les difficultés pour le domaine des grandeurs et mesures sont davantage liées aux méthodes d'apprentissage. Les liens entre grandeurs et mesures, système métrique et numération peuvent être abordés dès le début de la scolarité. La perception que ce domaine doit faire l'objet d'un apprentissage dans la durée depuis le cycle 1 n'est pas toujours identifiée par les PE.

La gestion de données et les grandeurs et mesures offrent des opportunités en termes de liens interdisciplinaires ou entre domaines mathématiques que mettent en évidence certains PE. Ces liens témoignent de la construction d'un rapport au savoir détaché d'un rapport en posture d'élève. Ces derniers constats soulèvent un paradoxe. Polyvalents, il reviendrait aux PE d'opérer une harmonisation interdisciplinaire, c'est-à-dire de lier par exemple gestion de données (lecture et interprétation de graphiques) et histoire (démographie). En réalité, pour certains, cette harmonisation n'est pas faite car des thèmes sont peu ou prou travaillés, peut-être en fonction du rapport au savoir des PE en posture d'élève (Magendie 2004). Par exemple, la lecture d'une pyramide des âges ne présente pas une réelle motivation pour les élèves de cycle 3 : « La pyramide des âges, je m'en fous quand j'ai 10 ans! » (PE5).

Cependant, le fait que nous ayons choisi, lors des entretiens, de ne pas questionner les PE sur le savoir mathématique scolaire à enseigner, peut expliquer que ceux-ci ont évoqué de manière privilégiée des savoirs transversaux.

\section{Troisième spécificité : leur rapport aux} mathématiques scolaires (qui peut légitimer le statut social du PE) présente dans leur propos tous les traits d'une conformité aux exigences institutionnelles éducatives et politiques (programme et socle commun)

D'un point de vue épistémique, les PE interrogés portent rarement un regard critique sur les programmes et leur interprétation les conduit à privilégier les domaines et activités où ils se sentent le plus à l'aise pour mobiliser leurs élèves (prévalence des petits problèmes concrets sur des problèmes plus complexes).

Ils n'identifient pas explicitement ce qui pourrait être novateur. Les concepteurs des programmes de 2008 ont souhaité une plus grande cohérence entre les apprentissages mais cet aspect est peu évoqué. Il s'agit, par exemple, de faire un lien plus explicite entre numération et système métrique (Chambris 
2010), d'introduire une procédure plus générale pour résoudre les problèmes de proportionnalité et d'exploiter la gestion de données avec d'autres disciplines. En l'absence de formation continue, l'appropriation de ces nouveaux enjeux est une charge parmi d'autres (la réforme ne concerne pas que les mathématiques).

Notre échantillon est constitué d'une proportion importante de maîtres formateurs. Pour 6 PE interrogés sur 9 , les pratiques déclarées sont les pratiques attendues institutionnellement d'un maître formateur impliqué dans la formation des professeurs stagiaires qu'il visite lors des stages et reçoit en formation dans sa propre classe. Il n'est donc pas étonnant que l'on trouve ici des pratiques déclarées conformes aux exigences institutionnelles. Des recherches complémentaires basées sur des observations en classe permettraient de prendre la mesure de l'écart entre les pratiques déclarées et les pratiques effectives.

Deuxième question de recherche : comment ces éventuelles spécificités influencent-elles les pratiques déclarées d'enseignement des mathématiques de ces professeurs?

Pour Mornata (2015, p. 76), « le rapport au savoir, dont la nature est éminemment sociale, se manifesterait par un contenu, des croyances sur le savoir et l'apprentissage, issu d'un processus de construction caractérisé par un investissement identitaire en lien avec le savoir $»$. Cet investissement identitaire, qui éclaire le rapport au savoir mathématique des PE, permet de faire le lien avec des pratiques déclarées et d'esquisser des traits communs ou distinctifs. Par pratiques déclarées, nous entendons les mathématiques que les PE disent enseigner, les démarches d'apprentissage dont ils usent, les ressources qu'ils mobilisent, c'est-à-dire, ce que nous avons analysé dans la dimension épistémique.

Nous retenons que, chez ces PE chevronnés, les aspects communs des pratiques évoquées peuvent trouver leur origine dans la spécificité de leur statut de PE. Le rapport au savoir mathématique scolaire est fortement marqué par leur rapport aux élèves et par le rapport aux injonctions officielles : il en résulte un consensus sur des pratiques sociales de référence qui renvoie plus largement à la dimension sociale de leur rapport à ce savoir spécifique. La variabilité des pratiques déclarées montre encore que la dimension identitaire modulée par une dimension sociale (les mathématiques pour moi, dans la société) impacte la dimension épistémique : c'est à l'éclairage de celle-ci que nous avons différencié ces pratiques. Et enfin, sous certaines conditions, ces pratiques peuvent évoluer...

\section{Traits communs des pratiques déclarées des PE interrogés}

D'un point de vue identitaire, nous avons relevé que les PE interrogés évoquent largement l'élève qu'ils ont été pour construire leur posture d'enseignant.

Dans les entretiens que nous avons réalisés, les enseignants ont souvent parlé de l'élève qu'ils se souviennent avoir été. Toutefois, nous ne rejoignons qu'en partie l'analyse de Magendie (2004, p. 111) qui souligne : "L'enseignant semble proposer (et permettre) à ses élèves des savoirs et des activités en adéquation avec le rapport à l'apprendre à l'école de l'élève qu'il pense avoir été ». Les PE chevronnés tels que ceux que nous avons interrogés mettent en ouvre la forme d'enseignement qu'ils estiment la plus appropriée à leurs élèves en adaptant leurs situations d'apprentissage en fonction des besoins de leurs élèves.

Nous avons aussi relevé que la dimension identitaire est modulée par la dimension sociale car les pratiques déclarées des PE sont en harmonie avec les valeurs de l'école.

Que les élèves s'apparentent à celui qu'ils ont été ou, au contraire, qu'ils leur semblent très différents, il s'agit pour les PE de construire un rapport au savoir qui concilie les besoins ressentis pour les élèves qu'ils ont en charge et les programmes. Leurs pratiques déclarées sont en résonance avec le sens et les valeurs du rapport au savoir mathématique scolaire véhiculé par l'école. Elles sont conformes aux pratiques attendues institutionnellement, sans doute en raison du nombre important de maîtres formateurs dans notre échantillon.

\section{Traits distinctifs des pratiques déclarées des PE interrogés}

Nous relevons différentes formes de rapport épistémique au savoir. 
Les trois formes du rapport épistémique au savoir mathématique des $\mathrm{PE}$, bien qu'imbriquées, sont identifiables à divers degrés chez les PE. Dans leur discours, trois enseignants (PE1-PE3-PE9) décrivent des savoirs, des pratiques, qui peuvent renvoyer à une certaine objectivation, un certain recul, par rapport au monde de l'action. Les enjeux de savoir sont premiers et congruents à des exigences institutionnelles intégrées; les ressources sont accessibles, mobilisables dans un contexte a priori transparent; c'est le processus épistémique nommé « objectivation-dénomination ». Pour d'autres (PE5-PE6), au-delà des enjeux de savoir, prime un rapport au savoir qui se livre dans l'expression de savoir-faire dans un contexte précisément évoqué (les élèves que j'ai en face de moi), de ressources nécessaires pour réguler le monde dans lequel j'enseigne; c'est le processus épistémique « imbrication du Je dans la situation ». En ce qui concerne le troisième processus "distanciation-régulation », il apparaît comme allant de soi dans les pratiques déclarées qui réfèrent toutes largement à la relation pédagogique. Pour les autres (PE2-PE4-PE7-PE8), les trois processus sont plus étroitement imbriqués.

En termes de dimension épistémique, nous avons dégagé deux profils de PE qui se distinguent par leur entrée par des savoirs spécifiques ou de manière plus globale.

PE1-PE3-PE9 peuvent présenter un rapport au savoir assez proche de celui des professeurs de lycée et collège car, pour eux, les savoirs mathématiques scolaires forment un tout structuré. Par exemple, pour PE3, le rapport au calcul est premier parce que « ciment» du contenu disciplinaire. La finalité des apprentissages est de construire des outils intellectuels. Les stratégies d'apprentissage sont axées sur les savoirs et leur épistémologie. Le PE est médiateur mais aussi expert; toutefois, il peut éprouver des difficultés à prendre en compte certains obstacles rencontrés par des élèves.

PE5-PE6 présentent un rapport au savoir qui accorde la priorité au développement global des compétences de l'élève. Ils ont une vision peut-être moins large de la cohérence des programmes; les exigences fixées par les programmes représentent surtout des outils pour se débrouiller dans la vie. Ils axent leurs stratégies d'apprentissage sur la mobilisation des élèves. Le PE est tuteur, expert à identifier et à prendre en compte les difficultés des élèves.

\section{Trajectoires en évolution}

La dimension identitaire semble piloter des trajectoires en évolution.

Rapport au savoir et pratiques déclarées ne sont pas figés. Ainsi, l'investissement identitaire consenti dans certaines trajectoires professionnelles peut modifier le rapport au savoir mathématique scolaire. Nous prendrons l'exemple de PE4. PE4 avait préparé le $\mathrm{CRPE}^{10}$ à l'IUFM où il a reçu une formation qui repose autant sur les compétences numériques que géométriques. Pour réussir le CRPE, PE4 a dû retravailler la géométrie démonstrative sélective et absente de l'école primaire. PE4 n'aimait pas enseigner la géométrie à l'école, prisonnier de ses souvenirs d'une géométrie face à laquelle il était en difficulté. Après une recherche-action sur la géométrie à l'école primaire, PE4 a vu son rapport au savoir géométrique en posture d'enseignant changer radicalement. Soutenues par ce travail collaboratif avec des chercheurs, ses pratiques se sont développées. D'un domaine mathématique détesté en tant qu'élève puis en tant qu'enseignant, cette expérience lui a permis d'évoluer vers un domaine qu'il prend maintenant plaisir à travailler avec ses élèves. A contrario, PE3, fasciné par les mathématiques, découvre les difficultés à mathématiser de ses élèves. Sans remettre en question son engagement, cela le contraint à prendre en compte les obstacles que rencontrent les élèves.

\section{CONCLUSION}

La question de l'articulation entre le rapport au savoir en mathématiques et l'engagement des PE à enseigner des mathématiques reste encore largement à explorer. Le travail conduit auprès d'enseignants chevronnés montre qu'il existe des conditions qui permettent aux PE de faire évoluer leurs pratiques. Si les modifications de programmes stricto sensu ne sont pas des vecteurs de transformation, les formations favorables aux échanges entre pairs, les recherches-actions qui prennent en compte les pratiques existantes (et donc le rapport au savoir des PE) apparaissent comme des leviers d'évolution. De nouvelles recherches sont nécessaires pour mettre à l'épreuve ces résultats sur un échantillon plus large et plus représentatif de PE mais aussi sur des pratiques effectives. 
Comment exploiter une meilleure connaissance $\mathrm{du}$ rapport au savoir en mathématiques des PE pour cibler les interventions les plus opportunes en formations initiale et continue? Nous rejoignons les conclusions de Maizières (2011, p. 11) qui préconise une formation professionnelle permettant « de questionner le sens et la valeur de la discipline au sein de l'éducation générale, avant même de donner les outils nécessaires pour répondre aux objectifs de cette éducation $»$. Tous les PE interrogés accordent du sens et de la valeur aux mathématiques scolaires. Ce que nous avons identifié, c'est que ce sens et cette valeur, liés à la trajectoire identitaire et professionnelle, peuvent différer même si la dimension sociale (au sens large, au service du développement de l'élève, au service de son intégration dans la société via la poursuite d'études) est un indicateur commun. C'est sur les diverses déclinaisons du sens et de la valeur que peut vraisemblablement prendre appui une formation adéquate. Par exemple, comment favoriser les liens entre les mathématiques et les autres disciplines scolaires? Chevallard (2003, p. 17) explique le résultat d'une évolution qui a conduit à la perte de sens aussi bien pour les élèves que pour les enseignants : "Le curriculum mathématique actuel est aujourd'hui marqué par un enfermement, une autarcie, voire un autisme disciplinaire qui sont l'aboutissement d'un siècle voué à la recherche véhémente de la pureté disciplinaire et au rejet corrélatif de tout métissage épistémologique ». Même si ce travers est moins lisible dans les programmes actuels, l'héritage demeure et reste présent comme le suggèrent les rapports au savoir de certains PE.

Par ailleurs, des opportunités existent. Tout d'abord, les nouveaux programmes de 2016 réorganisent les cycles en donnant davantage de poids à l'interdisciplinarité et au lien entre démarche de résolution de problèmes et démarche d'investigation. Ensuite, résolument orientées vers la pratique issue du terrain, les « lessons studies » sont intéressantes à plus d'un titre. Qu'elles visent à améliorer l'enseignement, à accompagner la mise en ouvre d'un nouveau programme national ou à intervenir dans le cadre de formations initiale et continue, les « lessons studies » consistent littéralement à étudier ce qui constituerait la leçon idéale pour des enseignants. Ce dispositif est axé autour d'une leçon ou d'une séquence précise, à concevoir, mettre en œuvre, puis à ouvrir à des collègues observateurs afin de permettre le partage des opinions. «Ainsi, il est capital que la leçon soit conçue, non pas comme la production de celui qui l'enseigne dans une classe particulière, mais comme un produit de l'équipe, produit que l'on est en train d'améliorer » Miyakawa et Winslow (2009, p. 82). Pour ces auteurs, ce dispositif constitue des « modèles explicites d'un processus d'ingénierie didactique en collectivité » dont le potentiel pour la formation des enseignants est prometteur (Miyakawa \& Winslow 2009, p. 85).

\section{NOTES}

1. Décret $n^{\circ} 2006-830$ du 11 juillet 2006 relatif au socle commun de connaissances et de compétences et modifiant le code de l'éducation. JORF $n^{\circ} 160$ du 12 juillet 2006 page 10396 texte $n^{\circ} 10$.

2. Quatre types de problèmes sont évoqués et peuvent être associés à des objectifs d'apprentissage différents :

- Problèmes dont la résolution vise la construction d'une nouvelle connaissance.

- Problèmes destinés à permettre le réinvestissement de connaissances déjà travaillées, à les exercer.

- Problèmes plus complexes que les précédents dont la résolution nécessite la mobilisation de plusieurs catégories de connaissances.

- Problèmes centrés sur le développement des capacités à chercher : en général, pour résoudre ces problèmes, les élèves ne connaissent pas encore de solution experte.

Dans ce dernier cas, nous parlerons de " problèmes pour chercher » alors que dans les précédents nous pourrions parler de « problèmes pour apprendre », en soulignant l'aspect réducteur de ces dénominations, puisque, dans tous les cas, l'élève mobilise des connaissances et se trouve placé en situation de recherche.

3. Nous noterons que les enseignants PE8 et PE9, qui n'ont pas actuellement en charge de classe en cycle 3, sont dans l'évocation...

4. PE4-PE5-PE6-PE7-PE8 (en gras) ont participé au protocole de recherche sur le rapport au savoir en physique des professeurs des écoles.

5. Ancienneté dans la fonction de PE.

6. La durée des entretiens varie entre 20 minutes et 1 heure 30 minutes.

7. Les questions posées ne renvoient pas à une unique sousdimension du rapport au savoir. Au contraire, les réponses recueillies renseignent souvent sur plusieurs « rapports à ». 8. Quelques questions posées pour renseigner la dimension sociale : 
- Que représentent les maths pour vous? Quel souvenir avez-vous de votre première rencontre avec les maths dans votre enfance? Avez-vous vécu une expérience traumatisante ave les mathématiques durant votre scolarité? Avez-vous le sentiment de faire ou utiliser des maths dans votre vie de tous les jours? Pourquoi faut-il faire des maths à l'école primaire? Liens avec d'autres disciplines?

9. Nous rappelons que les quatre domaines déclinés dans les Instructions Officielles de 2008 sont : 1) Nombres et calculs, 2) Géométrie, 3) Grandeurs et mesures, 4) Organisation et gestion de données.

10. Le CRPE est le Concours de Recrutement des professeurs des écoles.

\section{RÉFÉRENCES}

Ball, D.-L., Thames, M.-H. \& Phelps, G. (2008). Content Knowledge for Teaching. What Makes It Special? Journal of Teacher education, 59(5), 389-407.

Beillerot, J., Blanchard-Laville, C. \& Mosconi. N. (1996). Pour une clinique du rapport au savoir. Paris : L'Harmattan.

Caillot, M. (2014). Les rapports aux savoirs des élèves et des professeurs. In M.-C., Bernard, A., Savard \& C., Beaucher (dir.). Le rapport aux savoirs : une clé pour analyser les épistémologies enseignantes et les pratiques de classe (p. 7-18). Québec : CRIRES.

Calmettes, B. (2015). Des rapports aux savoirs aux rapports pragmatiques à l'enseigner. Valuation et action de l'enseignant. Analyses de cas relatifs aux démarches d'investigation, en physique, en France. In V. Vincent \& M.-F. Carnus (dir.), Le rapport au $(x)$ savoir(s) au coeur de l'enseignement : enjeux, richesse et pluralité (p. 87-99). Bruxelles : De Boeck.

Cappiello, P. \& Venturini, P. (2011). Usages de l'approche socio-anthropologique du rapport au savoir en sciences de l'éducation et en didactique des sciences : étude comparatiste. Carrefours de l'éducation, 2011/1 (31), 237-252.

Chambris, C. (2010). Relations entre grandeurs, nombres et opérations dans les mathématiques de l'école primaire au $20^{e}$ siècle : théories et écologie. Recherches en Didactique des Mathématiques, 30(3), 317-366.

Charlot, B. (1997). Du rapport au savoir, éléments pour une théorie. Paris : Anthropos.

Charlot, B. (2003). La problématique du rapport au savoir. In S. Maury \& M. Caillot (dir.), Rapport au savoir et didactiques (p. 33-50). Paris : Éditions Fabert.

Chevallard, Y. (2003). Approche anthropologique du rapport au savoir et didactique des mathématiques. Communication aux 3e Journées d'étude francoquébécoises (Université René-Descartes Paris 5, 17-18 juin 2002). In S. Maury \& M. Caillot (dir), Rapport au savoir et didactiques (p. 81-104). Paris : Éditions Fabert.

Decroix, A.-A., Chenevotot, F., Galisson, M.-G. \& Baheux, C. (2019). Comparaison des rapports au savoir en mathématiques et en physique de professeurs des écoles. In Abboud, M. (dir.), Mathématiques en scène, des ponts entre les disciplines, Actes du 76e colloque 'Espace Mathématique Francophone' (EMF2018 - GT1, 22-26 octobre 2018). Gennevilliers, France : Université de Cergy Pontoise - ESPE de l'Académie de Versailles.

Decroix, A.-A. \& Kermen, I. (2016). Rapport au savoir en physique-technologie des Professeurs des écoles. Neuvièmes rencontres scientifiques de l'ARDIST, Lens 30-31/03 et 1/04 2016.

d'Enfert, R. (2003). L'enseignement mathématique à l'école primaire de la Révolution à nos jours. Textes officiels tome I : 1794 -1914. INRP.

d'Enfert, R. (2015). L'enseignement mathématique à l'école primaire de la Révolution à nos jours. Textes officiels tome II : 1915- 2000. Pulim.

Develay, M. (1995). Apprentissage, enseignement, formation. Revue EPS 1(72), 2-6.

ERMEL (2000). Apprentissages numériques et résolution de problèmes. Paris : Hatier et INRP.

ERMEL (2006). Apprentissages géométriques et résolution de problèmes. Paris : Hatier et INRP.

Fayol, M. (2013). L'acquisition du nombre. Paris : Presses universitaires de France (Que sais-je ? 3941).

Houdement, C. (2003). La résolution de problèmes en question. Grand N, 71, 7-23.

Julo, J. (2002). Des apprentissages spécifiques pour la résolution de problèmes? Grand N, 69, 31-52.

Magendie, L. (2004). Rapport à l'apprendre et pratiques d'enseignement de professeurs d'école : études de cas en mathématiques. Mémoire de DEA, Université de Toulouse Le Mirail, Toulouse.

Maizières, F. (2011). Rapport au savoir musical des professeurs des écoles et pratiques d'enseignement : un transfert parfois complexe. Éducation \& Didactique, 5(2), 53-64.

Ministère de l'Éducation Nationale (2002). Bulletin Officiel hors-série $\mathrm{n}^{\circ} 1$ du 14 février 2002.

Ministère de l'Éducation Nationale (2005). Documents d'application des programmes. Mathématiques (brochure CNDP n 755A1155), février 2005.

Ministère de l'Éducation Nationale (2008). Bulletin Officiel hors-série $\mathrm{n}^{\circ} 3$ du 19 juin 2008.

Ministère de l'Éducation Nationale (2013). Bilan de la mise en œuvre des programmes issus de la réforme de l'école primaire de 2008. Rapport n²013-066.

Ministère de l'Éducation Nationale (2016). Direction de l'Evaluation, de la Prospective et de la Perspective. Note d'information $\mathrm{n}^{\circ} 33$ - Novembre 2016. TIMMS 2015 mathématiques et sciences. Évaluation internationale des élèves de CMl. 
Ministère de l'Éducation Nationale (2018). 21 mesures pour l'enseignement des mathématiques. Rapport remis le 12 février 2018 par Villani C. et Torossian C.

Miyakawa, T. \& Winsløw, C. (2009). Un dispositif japonais pour le travail en équipe d'enseignants : étude collective d'une leçon. Éducation E Didactique, 3(1), 77-90.

Mornata, C. (2015). Le rapport au savoir des enseignants : complémentarité des dimensions épistémiques, identitaires et sociales. In V. Vincent \& M.-F. Carnus (dir.), Le rapport $a u(x)$ savoir(s) au coeur de l'enseignement : enjeux, richesse et pluralité (p. 75-86). Bruxelles : De Boeck.

Passeron, J-C. \& Revel J. (2005). Penser par cas ; Raisonner à partir de singularités. In Penser par cas (p. 9-44). Paris : Éditions de l'EHESS.

Pautal, E., Venturini, P. \& Dugal, J.-P. (2008). Prise en compte du rapport aux savoirs pour mieux comprendre un système didactique. Un exemple en SVT à l'école élémentaire. Didaskalia, 33, 63-88.

Peltier, M.-L. (1995). La formation initiale, en mathématiques, des professeurs d'école: "entre conjecture et éternité », IREM Paris VII.

Therriault, G., Morel, M. \& Letscher (2015). Croyances épistémologiques et rapports aux savoirs de futurs enseignants de sciences et de sciences humaines : quelles relations avec les conceptions de l'enseignement et de l'apprentissage? In V. Vincent \& M.-F. Carnus (dir.), Le rapport au (x) savoir(s) au coeur de l'enseignement : enjeux, richesse et pluralité (p. 111-126). Bruxelles : De Boeck. 